\title{
VIGOROUS MOTIONS IN MAGMA CHAMBERS AND LAVA LAKES
}

\author{
By \\ Herbert E Huppert \\ and \\ M Grae Worster
}

IMA Preprint Series \# 862

August 1991 


\title{
VIGOROUS MOTIONS IN MAGMA CHAMBERS \\ AND LAVA LAKES
}

\section{HERBERT E HUPPERT* and M GRAE WORSTER ${ }^{\dagger}$}

\begin{abstract}
Magma chambers and lava lakes are storage reservoirs of molten rock, which gradually cools and solidifies after emplacement. The molten rock is a multicomponent silicate melt that crystallizes to form a solid whose components differ locally from those of the original melt. This difference in composition can drive strong convective motions in the melt and cause large-scale zonation of minerals.

This paper considers a series of general, one-dimensional problems in which a layer of a one- or two-component melt is cooled from above. We describe the fundamental fluid-mechanical processes involved, which include: the influence of compositional effects on the solidification; the formation of mushy layers - regions in which fluid and solid co-exist; and the kinetic effects associated with disequilibrium thermodynamics. The solutions of the resultant mathematical models are compared with data from laboratory experiments using aqueous solutions. The agreement between the two sets of results is shown to be very good. Calculations using the simple, geologically oriented, twocomponent anorthite-diopside system are presented and their geological interpretation discussed briefly.
\end{abstract}

1. Introduction. Many, if not most, investigations in geophysics involve solving inverse problems, though they are not always formulated as such. The continual appearance of inverse problems occurs because our measurements of the earth are mainly constrained to be taken on the surface, with only a few measurements, such as the gravity field and the magnetic field, taken from satellites. This means that our knowledge of the interior of the earth is gained almost entirely by inference rather than by pure deduction. However, before the concepts and techniques of inverse theory can be applied to any problem, it is necessary to have a complete understanding of solutions related to the direct problem.

This general statement is true for the particular investigations of the physical and chemical processes that occur in magma chambers, which are large storage reservoirs of molten rock that are supposed to exist a few kilometres beneath all volcanoes, of both the subaerial and submarine variety. No measurements have ever been made within a molten magma chamber, and are unlikely to be made in the foreseeable future. Our knowledge is thus based on measurements from the occasional outpourings from such chambers, as seen, for example, in figure 1, and from the theoretical concepts and calculations of the processes that are thought to occur within the chambers. One process of considerable importance in the temporal evolution of magma chambers is the slow transfer of heat from the chamber to the surrounding country rocks and the resulting solidification that occurs. In order to investigate some aspects of this evolution, the current paper presents the solutions to a series of one-dimensional problems describing the solidification of an initially fluid layer due to cooling that

*Institute of Theoretical Geophysics, University of Cambridge, 20 Silver Street, Cambridge CB3 $9 \mathrm{EW}$, UK. The work of the first author is partially supported by a grant from Venture Research International.

${ }^{\dagger}$ Department of Engineering Sciences \& Applied Mathematics, and Department of Chemical Engineering, Northwestern University, Evanston Illinois 60208, USA 
takes place at the top of the layer. We sequentially build up the fundamental concepts and then apply them in order to discuss quantitatively the thermodynamic and fluid dynamic processes that occur in magma chambers.

The simplest situation to analyse, in which a one-component, or pure, melt solidifies, is known as the Stefan problem [1,2]. A semi-infinite layer of liquid, which is initially at a uniform temperature, is suddenly subjected at its base to a maintained temperature that is below the solidification temperature. Because the subsequent temperature in the liquid increases with height above the base it is assumed that no fluid motion occurs. The (one-dimensional) temperature profile in both the liquid and the resulting solid is determined by solving time-dependent diffusion equations in both media, possibly with a different coefficient of thermal diffusion in each layer. Because there is no length or time scale specified by the problem, the solution can be expressed in terms of a similarity variable and the solid phase grows in thickness at a rate proportional to $t^{1 / 2}$, where $t$ is the time from the initiation of the temperature at the base.

Cooling a layer of pure melt from above, rather than from below, leads to a temperature profile that increases with depth and hence, in most cases, is thermally unstable. This generally results in fluid motions, in the form of convection, whose vigour is determined by the Rayleigh number based on the (finite) depth of the liquid layer remaining at any time. This situation was first analysed by Turner, Huppert and Sparks [3] who explained all the essential features of the motion and evaluated the rate of solid production. However, their analysis was presented in an appendix whose primary aim was to aid the explanation of a series of experiments in which twocomponent melts were solidified. In addition, the subject and its interpretation has advanced since that time and the results of the calculations have been occasionally misinterpreted. It thus seems sensible to present a new, careful description of the problem in Section 2. We will also explain there what general principles can be learnt from the analysis about the fluid behaviour and the solidification processes in lava lakes.

In Section 3, we commence a discussion of the influence of compositional effects on both the solidification processes and the resulting fluid motions. We consider the cooling from above of a layer of a two-component melt that releases less dense fluid upon solidification. As solidification proceeds, a mushy layer develops, which consists of sparse interconnected solid with interstitial melt. Our initial analysis of this mushy layer, following Kerr et al. [4,5], considers the solidification to take place under strict thermodynamic equilibrium. In Section 4 we replace this constraint by one that incorporates kinetic effects into the solidification process [6]. Possibly the most important conclusion of this section is that vigorous convective motions can still result in the fluid even though there is no initial superheat; that is, even though the fluid lies initially on the liquidus and carries no additional thermal energy.

We quantitatively consider the consequences of this conclusion, and the other results that are derived from the model, for the two-component system made up of anorthite and diopside in Section 5. This simple system can be considered to have 
convects vigorously, the conservation relationship becomes ${ }^{1}$

$$
\left.k_{s} \frac{\partial T}{\partial z}\right|_{s-}=F_{T}+\left[c_{m}\left(T_{m}-T_{s}\right)+\mathcal{L}\right] \frac{d s}{d t},
$$

where

$$
F_{T}=\gamma k_{m}\left(\alpha g / \kappa_{m} \nu\right)^{1 / 3}\left(T_{m}-T_{s}\right)^{4 / 3}
$$

$k_{s}$ and $k_{m}$ are the thermal conductivities of the solid and the melt respectively, $F_{T}$, the thermal flux from the vigorously convecting fluid to the interface, is given by the oft-used four-thirds law (2.3) [7], $c_{m}$ is the specific heat per unit volume of the melt, $T_{m}$ is the mean temperature of the melt, $\mathcal{L}$ is the latent heat released as melt transforms to unit volume of solid, $\gamma$ is an empirical constant with a value of $0.14, \alpha$ is the coefficient of thermal expansion of the melt, $g$ is the acceleration due to gravity, $\kappa_{m}$ is the thermal diffusivity of the melt and $\nu$ is the viscosity of the melt, which is assumed to be constant. The assumption has also been made that any change in volume upon solidification can be neglected. The thermal flux $F_{T}$ leads to a decrease in temperature of the melt layer which is expressed by

$$
F_{T}=-c_{m}(H-s) \frac{d T_{m}}{d t}
$$

The conductive flux which appears on the left-hand-side of (2.2) needs to be evaluated from the solution of the diffusion equation in the solid. Alternatively, on the assumption that the thickness of the solid $s(t)$, which is governed by the release of latent heat, is very much less than the scale of thermal diffusion in the solid, $\left(\kappa_{m} t\right)^{1 / 2}$, the temperature profile in the solid is very nearly linear and

$$
\left.T_{z}\right|_{s-}=\left(T_{s}-T_{A}\right) / s
$$

The mathematical problem thus consists of solving (2.2)-(2.5) together with the initial conditions

$$
T_{m}=T_{0}, \quad s=0 \quad(t=0)
$$

It is convenient at this stage to define dimensionless variables by

$$
u=\left(T_{m}-T_{s}\right) /\left(T_{0}-T_{s}\right), \quad v=s / H \quad \text { and } \quad \tau=\kappa_{m} t / H^{2},
$$

where time has been non-dimensionalised by the conductive time scale, $H^{2} / \kappa_{m}$. The variable $u$ represents the remaining superheat of the melt, while $v$ represents the fraction of the layer solidified. Substituting (2.7) into (2.2)-(2.6), we obtain

$$
\frac{1}{v}=\mathrm{Q} \mathcal{N} u^{4 / 3}+(\mathrm{Q} u+\tilde{\mathcal{S}}) \frac{d v}{d \tau}
$$

\footnotetext{
${ }^{1}$ The term involving $c_{m}$ on the right-hand side of (2.2) was inadvertently omitted in [3]; however, in most cases it plays a minor role. Further details of the differences between the current presentation and that in [3] are discussed in footnote " 2 ".
} 


$$
(1-v) \frac{d u}{d \tau}=-\mathcal{N} u^{4 / 3}
$$

and

$$
u=1 \quad v=0 \quad(\tau=0),
$$

in terms of the three dimensionless parameters that describe the system

$$
\mathrm{Q}=\frac{k_{m}}{k_{s}} \frac{\left(T_{0}-T_{s}\right)}{\left(T_{s}-T_{A}\right)}
$$

a modified Stefan number

$$
\tilde{\mathcal{S}}=\frac{k_{m}}{k_{s}} \frac{\mathcal{L}}{c_{m}\left(T_{s}-T_{A}\right)}
$$

and the initial Nusselt number for the melt

$$
\begin{aligned}
\mathcal{N} & =\gamma\left[\alpha g\left(T_{0}-T_{s}\right) H^{3} / \kappa_{m} \nu\right]^{1 / 3} \\
& \equiv \gamma \mathrm{Ra}_{\text {init }}^{1 / 3},
\end{aligned}
$$

where $R a_{\text {init }}$ is the initial Rayleigh number of the system. The physical interpretation of the governing equation (2.8) is that the conductive heat flux through the solid balances the three terms on the right-hand side of (2.8) which represent respectively: the convective flux that is transferred from the melt; the sensible heat that is lost as the melt is cooled to its solidification point; and the latent heat that is released upon solidification.

In general, solutions to the non-linear coupled set of equations (2.8)-(2.10) can only be obtained numerically. However, useful approximate analytical solutions can be derived in the geologically relevant situation of $\mathcal{N} \gg 1$, with $\mathrm{Q}$ and $\tilde{\mathcal{S}}$ fixed. The analysis is based on the principle that, since each term in (2.8) is positive, the term on the left-hand side is always important. Initially, because of the singularity of this term, as indicated by $(2.10 \mathrm{~b})$, it is balanced by the last two of the three terms that make up the right-hand side of (2.8). As $\tau$ increases and the rate of advance of the interface $d v / d \tau$ decreases, the first term of (2.8) becomes important. Later, its influence wanes as the superheat $u$ decays, and the balance of terms returns to the initial one.

In physical terms, this variation is expressed as: an initial balance between a large conductive flux through the thin solid phase and latent heat release; a period during which the convective flux from the interior exceeds the latent heat release and retards growth of the solid; and a final period during which latent-heat effects again overpower the dwindling convective flux. The analysis below demonstrates that the initial phase occurs for $0<\tau<O\left(\mathcal{N}^{-2}\right)$, the intermediate stage for $O\left(\mathcal{N}^{-2}\right)<\tau<O\left(\mathcal{N}^{-6 / 7}\right)$ and the final stage for $O\left(\mathcal{N}^{-6 / 7}\right)<\tau<\tau_{s}$, where $\tau_{s}$ is the (dimensionless) time, of order unity, for total solidification to occur. 
The analysis proceeds in three stages. In the first, at early times, (2.8)-(2.10) can be approximated as

$$
\begin{aligned}
& \frac{1}{v}=(\mathrm{Q} u+\tilde{\mathcal{S}}) \frac{d v}{d \tau} \\
& \frac{d u}{d \tau}=0
\end{aligned}
$$

$$
v=0, \quad u=1 \quad(\tau=0),
$$

the solution to which is

$$
v=\left(\frac{2}{\mathrm{Q}+\tilde{\mathcal{S}}}\right)^{1 / 2} \tau^{1 / 2} \quad \text { and } \quad u=1 .
$$

This solution is valid until

$$
\begin{aligned}
\mathrm{QN} & \sim(\mathrm{Q}+\tilde{\mathcal{S}}) \frac{d v}{d \tau} \\
& =\left(\frac{\mathrm{Q}+\tilde{\mathcal{S}}}{2}\right)^{1 / 2} \tau^{-1 / 2}
\end{aligned}
$$

that is, until

$$
\tau \sim \frac{\mathrm{Q}+\tilde{\mathcal{S}}}{2 \mathrm{Q}^{2}} \mathcal{N}^{-2}
$$

at which time

$$
v \sim(\mathrm{Q} \mathcal{N})^{-1} \ll 1 .
$$

For a while thereafter, the first term dominates the other terms of the right-hand side of (2.8), and equations (2.8) and (2.9) can then be written as

$$
\frac{1}{v}=\mathrm{QN} u^{4 / 3}
$$

$$
\frac{d u}{d \tau}=-\mathcal{N} u^{4 / 3}
$$

with matching to (2.8) giving the initial condition

$$
u(0)=1
$$

to leading order. The solution of (2.19)-(2.21) can be readily obtained as

$$
u=\left(1+\frac{1}{3} \mathcal{N} \tau\right)^{-3} \quad \text { and } \quad v=(\mathrm{Q} \mathcal{N})^{-1}\left(1+\frac{1}{3} \mathcal{N} \tau\right)^{4} .
$$

This solution remains valid until

$$
\mathrm{Q} \mathcal{N} u^{4 / 3} \sim \tilde{\mathcal{S}} \frac{d v}{d \tau},
$$


with $u$ and $v$ given by (2.22). That is, until

$$
\tau \sim\left(\frac{3 Q^{2}}{4 \tilde{\mathcal{S}}}\right)^{\frac{1}{7}} \mathcal{N}^{-\frac{6}{7}}
$$

At this time

$$
u \sim\left(\frac{3 \mathrm{Q}^{2}}{4 \tilde{\mathcal{S}}}\right)^{-\frac{3}{7}} \mathcal{N}^{-\frac{3}{7}} \text { and } \quad v \sim \frac{1}{\mathrm{Q}}\left(\frac{3 \mathrm{Q}^{2}}{4 \tilde{\mathcal{S}}}\right)^{\frac{4}{7}} \mathcal{N}^{-\frac{3}{7}}
$$

In the short period of time given by (2.24), convection causes the superheat $u$ to decay to the small value given by $(2.25 \mathrm{a})$ while little solid has grown, as indicated by $(2.25 b)$.

In the final stage, the thermal balance is similar to that expressed in equation (2.8), except that now $u \sim 0$ to leading order. Thus

$$
\frac{1}{v}=\tilde{\mathcal{S}} \frac{d v}{d \tau}
$$

while equation (2.9) is most conveniently written in the form

$$
\frac{1-v}{v} \frac{d u}{d v}=-\mathcal{N} u^{\frac{4}{3}}
$$

The solution to equations (2.26) and (2.27) that matches with the solutions at earlier times is given by

$$
v=\left(\frac{2}{\tilde{\mathcal{S}}}\right)^{\frac{1}{2}} \tau^{\frac{1}{2}} \quad \text { and } \quad u=\left[1-\frac{1}{3} \mathcal{N} \tilde{\mathcal{S}}(\ln (1-v)+v)\right]^{-3}
$$

Note that this asymptotic expression for $u$ is uniformly valid for all times ${ }^{2}$.

Some results of integrating equations (2.8)-(2.10) numerically are shown in figures 3 and 4. The general behaviour of this system of equations is best seen in figure 3 , for which the parameter values used were $\tilde{\mathcal{S}}=1, \mathrm{Q}=1$, and $\mathcal{N}=100$. This value of $\mathrm{Q}$ is much larger than would typically apply to magma chambers but the values chosen here serve to illustrate the structure of the solutions. The curve for $v$ in figure $3 \mathrm{a}$ has two points of inflexion, corresponding to the transitions between the three stages of evolution represented by $(2.15),(2.22)$, and (2.28). These transitions can be seen more easily. in figure $3 \mathrm{~b}$ in which $\log v$ is plotted as a function of $\log \tau$. The transition times of $\mathrm{O}\left(\mathcal{N}^{-2}\right)$ and $\mathrm{O}\left(\mathcal{N}^{-6 / 7}\right)$ are indicated with dashed lines. Note that $v$ increases like $\tau^{1 / 2}$ in both the first and third stages of evolution. During the second stage of evolution, the large convective heat flux from the melt serves to slow down the rate of growth until the superheat has decayed to small values and the convective

\footnotetext{
${ }^{2}$ Most of the solutions obtained in the current presentation have their antecedents in the solutions derived in [3]. The two main differences in the presentations are, first, the different nondimensionalizations of time that are employed, and second, that, in [3], solutions for different ranges of the dimensionless parameters are discussed, whereas, in the current paper, we consider all the parameters to be fixed and determine the temporal evolution of the solutions under the assumption that $\mathcal{N} \gg 1$.
} 

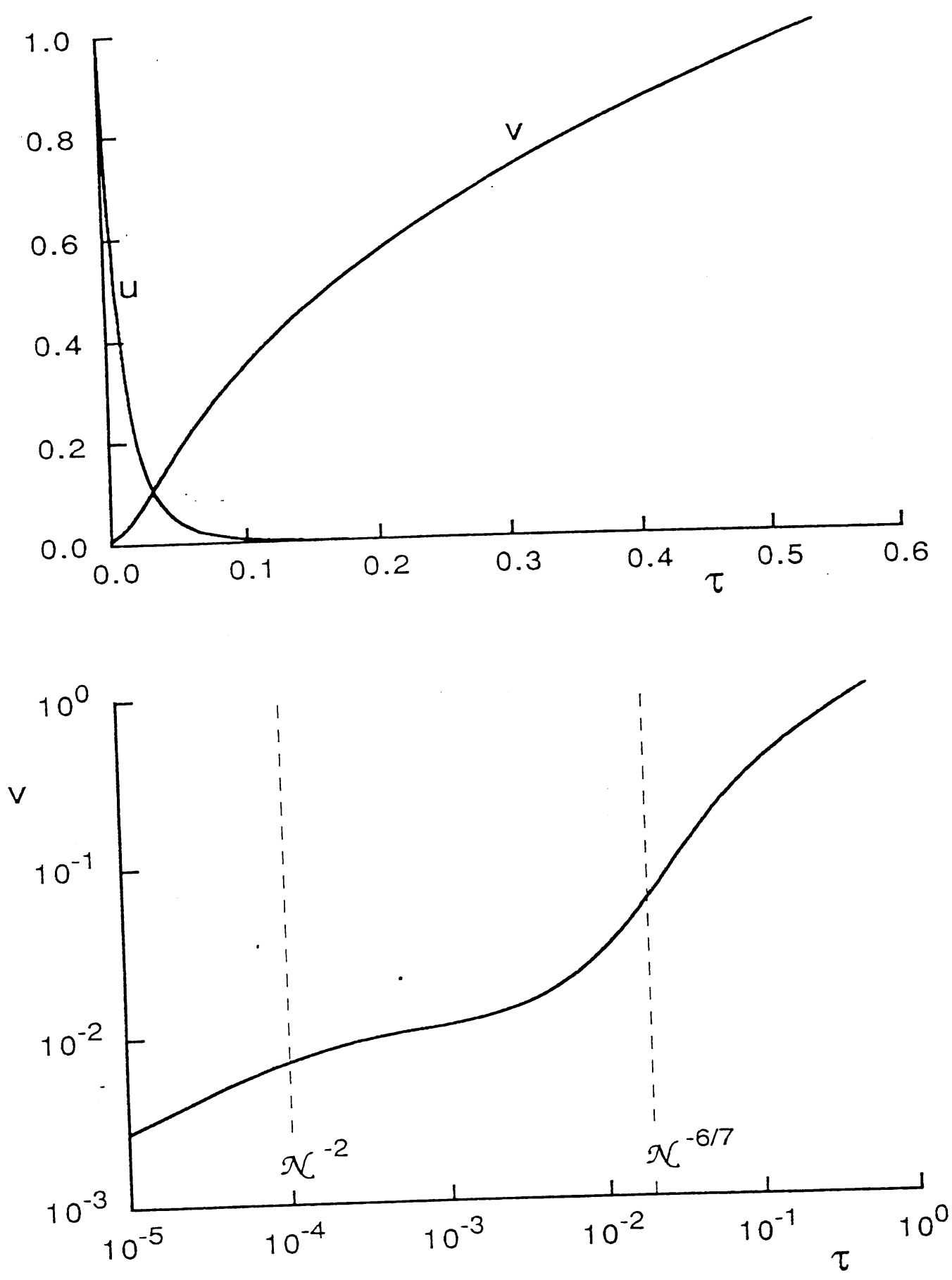

Figure 3: Results of numerical calculations of the solidification of a layer of liquid cooled from above using dimensionless parameter values of $\tilde{\mathcal{S}}=1, \mathcal{N}=100$, and $\mathrm{Q}=1$. (a) The dimensionless temperature of the melt $u$ and the depth of solid at the roof $v$ as functions of the dimensionless time $\tau$. (b) The depth of the solid layer as a function of time shown on logarithmic axes to illustrate the different stages of evolution. The vertical dashed lines indicate the approximate transition times as determined by the asymptotic analysis in section 2 . 

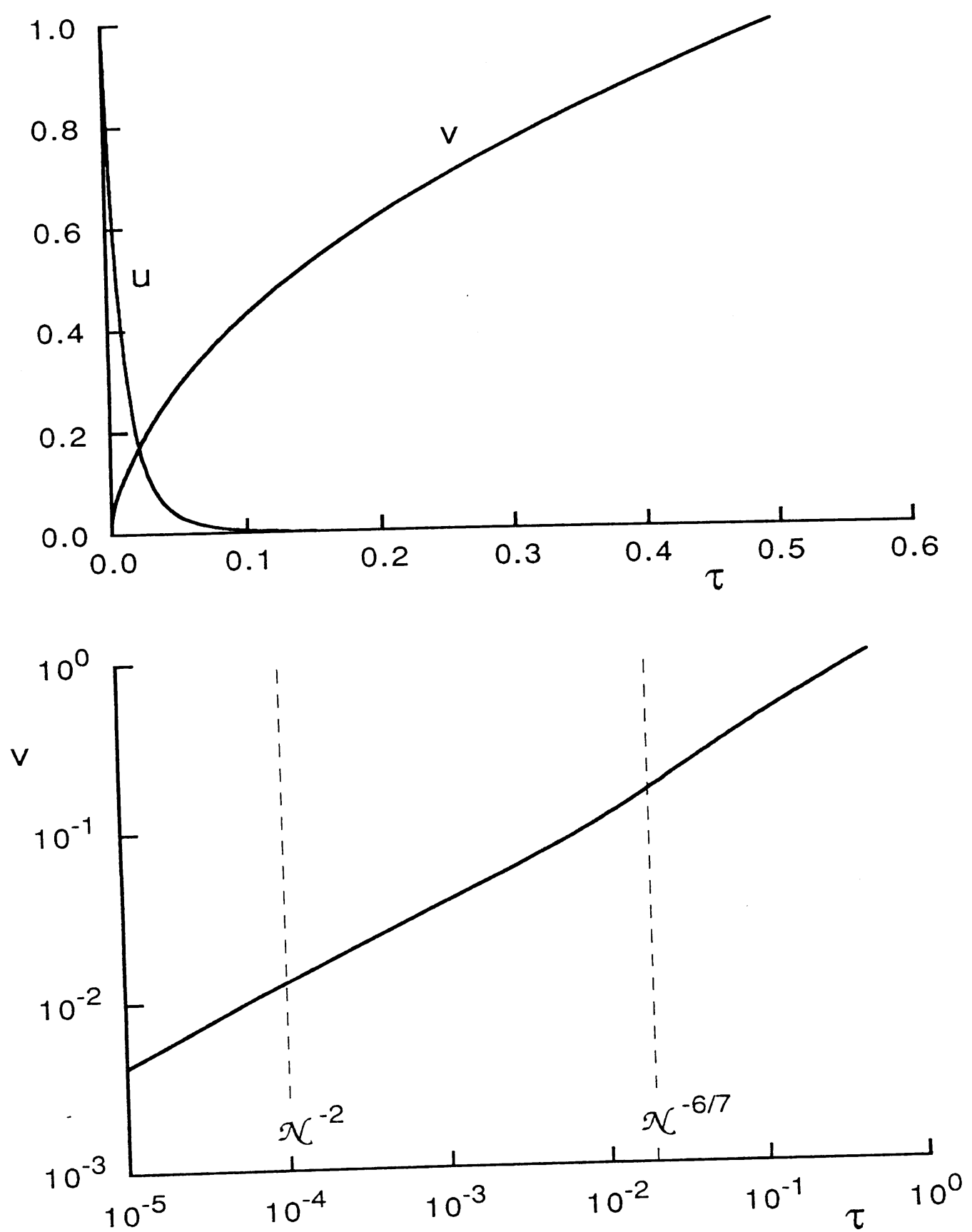

Figure 4: Results of numerical calculations of the solidification of a layer of liquid cooled from above using dimensionless parameter values of $\tilde{\mathcal{S}}=1, \mathcal{N}=100$, and $\mathrm{Q}=0.1$. (a) The dimensionless temperature of the melt $u$ and the depth of solid at the roof $v$ as functions of the dimensionless time $\tau$. (b) The depth of the solid layer as a function of time shown on logarithmic axes to illustrate the different stages of evolution. The vertical dashed lines indicate the approximate transition times as determined by the asymptotic analysis in section 2 . Note that, in contrast to figure $2 \mathrm{~b}$, the growth is approximately proportional to $\tau^{1 / 2}$ for all time. This is because the dimensionless initial superheat is smaller in this case, which causes the convection to have less influence on the solidification at the roof. 


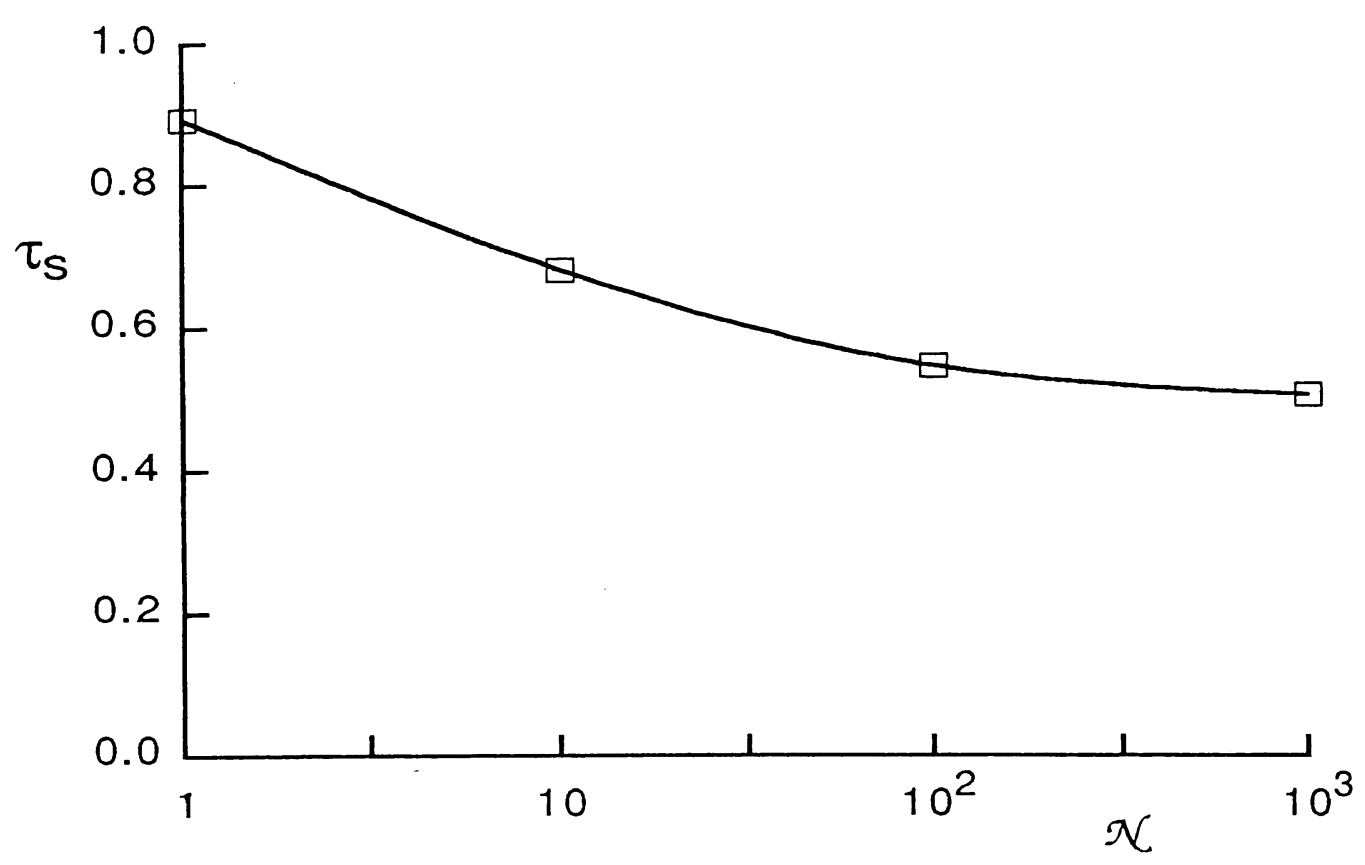

Figure 5: The dimensionless time $\tau_{s}$ for complete solidification as a function of the initial Nusselt number $\mathcal{N}$. Note that, as the convective intensity increases, the solidification time decreases relative to the timescale for thermal diffusion.

heat flux has diminished. The overall effect of convection, however, is to remove the superheat rapidly and thus to allow the system to solidify more quickly. Figure 3a shows the superheat $u$ decaying rapidly to small values by the end of the second stage of evolution. Note, however, that this decay is only algebraic and that the Rayleigh number based upon these small values of the superheat remains large until dimensionless times of order unity.

Figure 4 shows the results obtained with parameter values of a size appropriate to magma chambers. In particular, the dimensionless initial superheat $Q$ was taken to be only 0.1 , while the other parameter values were kept the same as used for figure 3. We again see the superheat $u$ decaying rapidly in figure $4 \mathrm{a}$. Now, however, although the Rayleigh number based upon the value of the superheat is still very large, the convective heat flux is always small in comparison with the heat flux conducted through the roof, and the growth of $v$ is almost proportional to $\tau^{1 / 2}$ for all time, as seen in figure $4 \mathrm{~b}$. Convection still plays a role in reducing the time for complete solidification, however, as can be seen in figure 5 , which shows $\tau_{s}$ as a function of the initial Nusselt number $\mathcal{N}$.

The analysis above can be considered as an approximate model for the evolution of a lava lake. These form when lava from an eruption flows into a depression in the earth's surface. The lakes may be a few meters to a few tens of meters deep $[8,9]$ and are cooled by contact with the atmosphere at the top surface. There are three major implications of the model just presented when the results are applied to a lava lake. The first is that any superheat is removed rapidly. It then follows that the lake solidifies in a time comparable to the conductive time scale $H^{2} / \kappa$. Finally, during this time, vigorous convective motions will take place in the interior of the lake. All three 
of these conclusions are in broad agreement with field data obtained from lava lakes, and remain valid even as we add more detail to the model in the following sections.

Further discussion of lava lakes is deferred until section 6 .

3. Compositional effects. When the melt that is being cooled and solidified is composed of more than one pure component, various interesting additional phenomena occur. In general, the solid product is of a different composition from the melt. Thus compositional gradients are generated in the melt as it becomes locally depleted of the components forming the solid phase. The compositional gradients often result in density gradients that can drive convection. These same gradients of composition can be the cause of morphological instabilities of the solid-liquid interface that ultimately result in the formation of a mushy zone separating the completely solid region from the melt (see figure 6). Mushy zones can also be anticipated from the equilibrium phase diagram of a multi-component system. Whereas there is a single temperature (the melting temperature) at which a pure solid is in equilibrium with its melt, there is a range of temperatures in which solid can coexist in equilibrium with a multi-component melt. This range is bounded by the solidus temperature, below which the system is completly solid, and the liquidus temperature, above which the system is completely liquid. These temperatures as functions of composition are often plotted to form the equilibrium phase diagram for the multi-component system that shows which phases are in equilibrium in a sample of given uniform temperature and bulk composition. The particular phase diagram for the anorthite-diopside system is shown in figure 7, which is also a typical phase diagram for many simple two-component systems that freeze to form an almost pure solid (i.e. the solidus curves are vertical). Between the solidus and liquidus temperatures, solid and liquid typically coexist in intimate contact, such that the microstructure has a lengthscale that is very small compared with the lengthscale of the mushy layer as a whole. This fact is the basis for our mathematical modelling of the mushy region.

In this paper, we restrict attention to systems that are cooled from above and which leave a buoyant residual as solid is formed from the melt. Since the effect of compositional variations upon the density of the liquid is typically much greater than the effect of the associated temperature variations within mushy regions, the interstitial fluid within the mushy layer that forms near the cooled roof is stably stratified and we assume therefore that it is stagnant. The model presented here was developed in reference [5], where a careful and detailed account of its assumptions and conditions can be found.

The two-component system that we consider first is similar in many ways to the single-component system analysed in section 2 . The solid region of figure 2 is replaced by a mushy region in figure 6 . The region of melt is modelled in the same way and its temperature obeys equation (2.4) with the heat flux given by equation (2.3), except that the solidification temperature $T_{s}$ is replaced by the (unknown) temperature $T_{i}$ of the interface between the mushy and liquid regions. If the diffusivity of solute $D$ in 


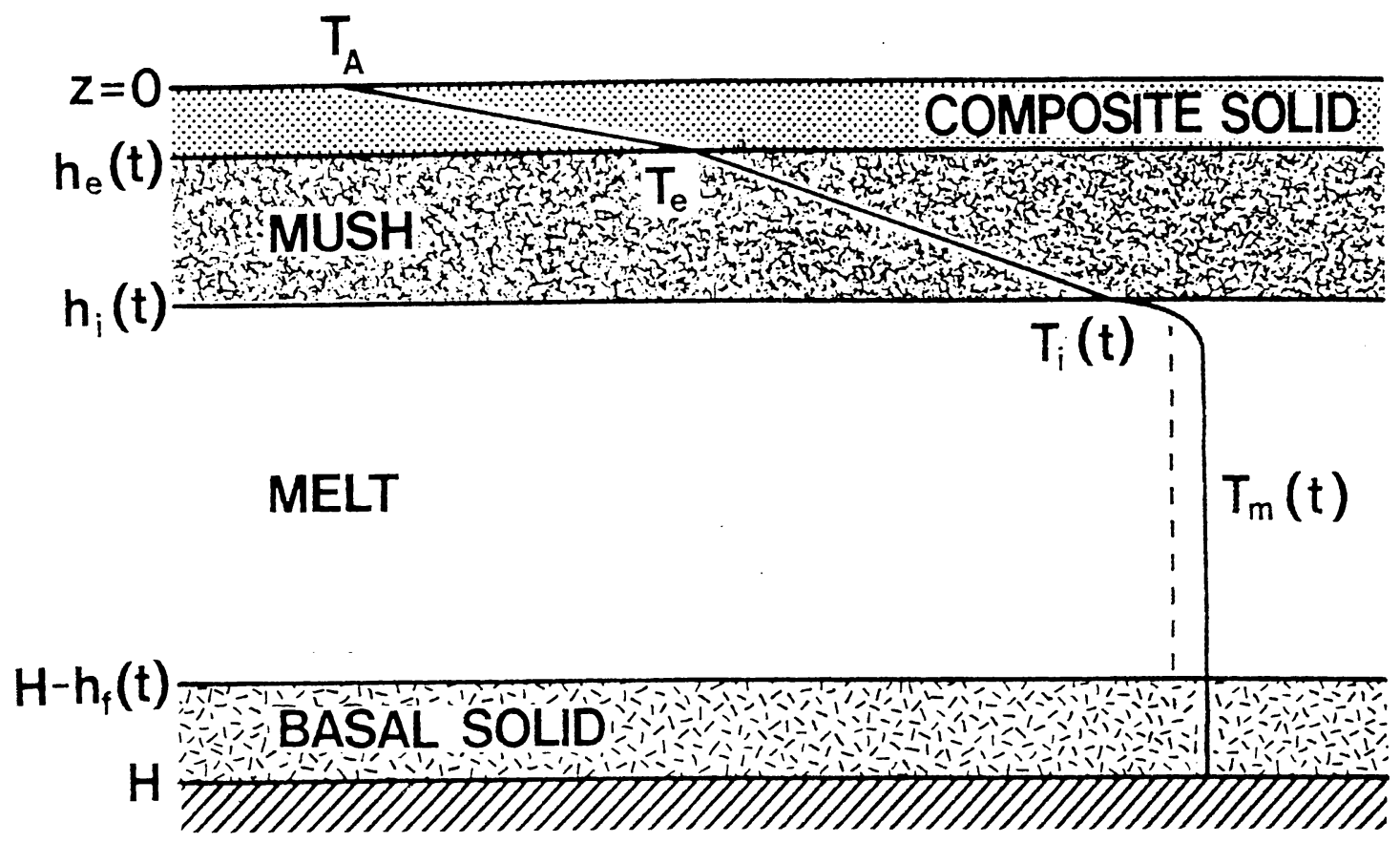

Figure 6: A schematic diagram of a solidifying layer of binary melt that is cooled from above. A mushy layer comprising interconnected crystals and interstitial liquid grows from the melt near the roof. If the temperature of the cooled roof is lower than the eutectic temperature then a completely solid, composite layer forms adjacent to the roof, comprising crystals of both end members of the binary system. A layer of crystals is observed to form near the insulated base in laboratory experiments and is predicted by the theory once the kinetic undercooling at the mush-liquid interface is taken into account.

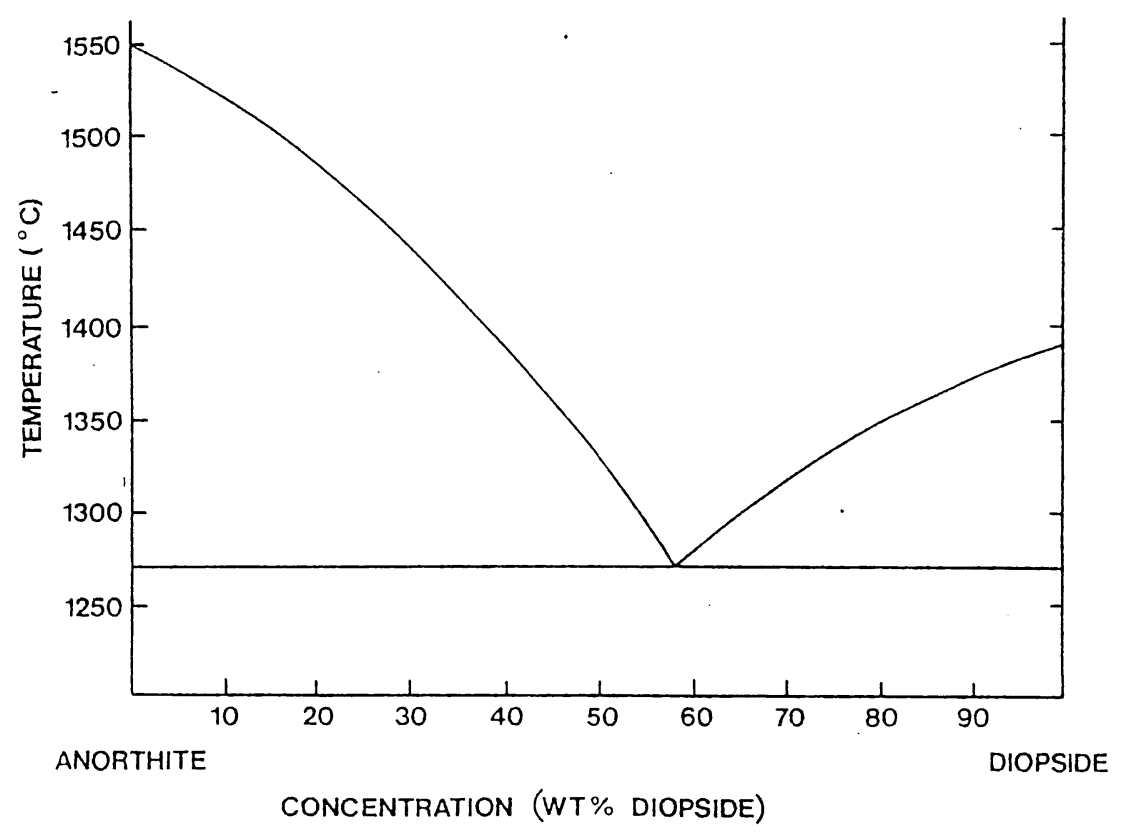

Figure 7: The equilibrium phase diagram for the anorthite-diopside system. The eutectic temperature of $1274^{\circ} \mathrm{C}$ is attained on the liquidus curve at a concentration of $58 \mathrm{wt} \%$ diopside. 
the melt is small compared with the diffusivity of heat $\kappa$, as is typically the case, then the compositional boundary layer ahead of the mush-liquid interface is negligible and the interfacial temperature is given by

$$
T_{i}=T_{L}\left(C_{m}\right)
$$

where $T_{L}(C)$ is the liquidus temperature of the two-component system and $C_{m}$ is the composition of the melt. Equation (3.1) is equivalent to equation (2.1) and is appropriate under the assumption of local equilibrium. In section 4 we shall replace equation (3.1) with a non-equilibrium growth law and discover important consequences of disequilibrium. For now we use the simpler condition of equilibrium thermodynamics in order to focus attention on effects related solely to having a multi-component melt, rather than a single-component melt.

The equation expressing conservation of heat at the mush-liquid interface (2.2) is replaced by

$$
\left.k_{m} T_{z}\right|_{h_{i}-}=F_{T}+\left(T_{m}-T_{i}\right) \frac{d h_{i}}{d t}
$$

where $z=h_{i}$ is the position of the mush-liquid interface. Note that there is no release of latent heat at this interface since the solid fraction in the mushy layer there is zero when $D / \kappa \ll 1$ and thermodynamic equilibrium is assumed [5,11]. Instead, the release of latent heat is distributed throughout the mushy layer as expressed by the thermal-diffusion equation, which now takes the form

$$
\bar{c} \frac{\partial T}{\partial t}=\frac{\partial}{\partial z}\left(\bar{k} \frac{\partial T}{\partial z}\right)+\mathcal{L} \frac{\partial \phi}{\partial t} .
$$

The release of latent heat is proportional to the rate of change of the solid fraction $\phi$ within the mushy layer, while the mean thermodynamic properties of the layer are functions of $\phi$, which are taken to be

$$
\begin{aligned}
& \bar{c}=\phi c_{s}+(1-\phi) c_{m}, \\
& \bar{k}=\phi k_{s}+(1-\phi) k_{m} .
\end{aligned}
$$

Note that equation (3.4a) is exact, while equation (3.4b) is an approximation that has led to good results in earlier studies $[4,10,11]$. An additional equation is required in order to determine how the solid fraction varies within the mushy layer. Since the microstructure of the mushy layer is so fine and the relative surface area of phase boundaries within the layer is consequently so large, it is a very good approximation to assume that

$$
T=T_{L}(C)
$$

within the layer; that is, the mushy region is considered to be in local thermodynamic equilibrium. The concentration $C$ of the interstitial liquid is found by conservation of solute to satisfy

$$
(1-\phi) \frac{\partial C}{\partial t}=\left(C-C_{s}\right) \frac{\partial \phi}{\partial t}
$$


where we have ignored the diffusion of solute.

Equations (3.1)-(3.6), together with equations (2.3) and (2.4), can be conveniently combined into the dimensionless system of equations and boundary conditions [5]

$$
\begin{gathered}
c \frac{\partial \theta}{\partial t}=\frac{\partial}{\partial z}\left(k \frac{\partial \theta}{\partial z}\right) \quad\left(0 \leq z \leq h_{i}\right), \\
\theta=-1 \quad(z=0), \\
\theta=0 \quad\left(z=h_{i}\right),
\end{gathered}
$$

where

$$
\begin{aligned}
& k \equiv \phi \frac{k_{s}}{k_{n}}+1-\phi, \\
& c \equiv \phi \frac{c_{s}}{c_{m}}+1-\phi+\frac{\mathcal{S}}{\mathcal{C}}(1-\phi)^{2},
\end{aligned}
$$

and

$$
\phi \equiv \frac{-\theta}{\mathcal{C}-\theta}
$$

with

$$
\begin{gathered}
\theta_{m} \dot{h}_{i}=\left.\frac{\partial \theta}{\partial z}\right|_{h_{i}-}-\tilde{\mathcal{N}} \theta_{m}^{\frac{4}{3}}, \\
\left(1-h_{i}\right) \dot{\theta}_{m}=-\tilde{\mathcal{N}} \theta_{m}^{\frac{4}{3}}, \\
h_{i}=0, \quad \theta_{m}=\theta_{0} \quad(t=0) .
\end{gathered}
$$

In these dimensionless equations, lengths and time have been scaled as in section 2, while the dependent variable $\theta$ is defined by

$$
0=\frac{T-T_{L}\left(C_{0}\right)}{T_{L}\left(C_{0}\right)-T_{A}}
$$

Note that, here, the temperature has been scaled with the temperature difference across the solidifying region, rather than that across the thermal boundary layer in the melt as it was in section 2. Consequently, the parameter in equations (3.9) and (3.10) is $\tilde{\mathcal{N}}=\theta_{0}^{-1 / 3} \mathcal{N}$, where $\mathcal{N}$ is the initial value of the Nusselt number, and should not itself be misinterpreted as a Nusselt number. A new parameter is introduced in these equations,

$$
\mathcal{C}=\frac{C_{s}-C_{0}}{C_{0}-C_{A}},
$$

where $T_{L}\left(C_{A}\right)=T_{A}$, which represents the difference in composition between the solid and liquid phases relative to the variations in concentration of the liquid phase 


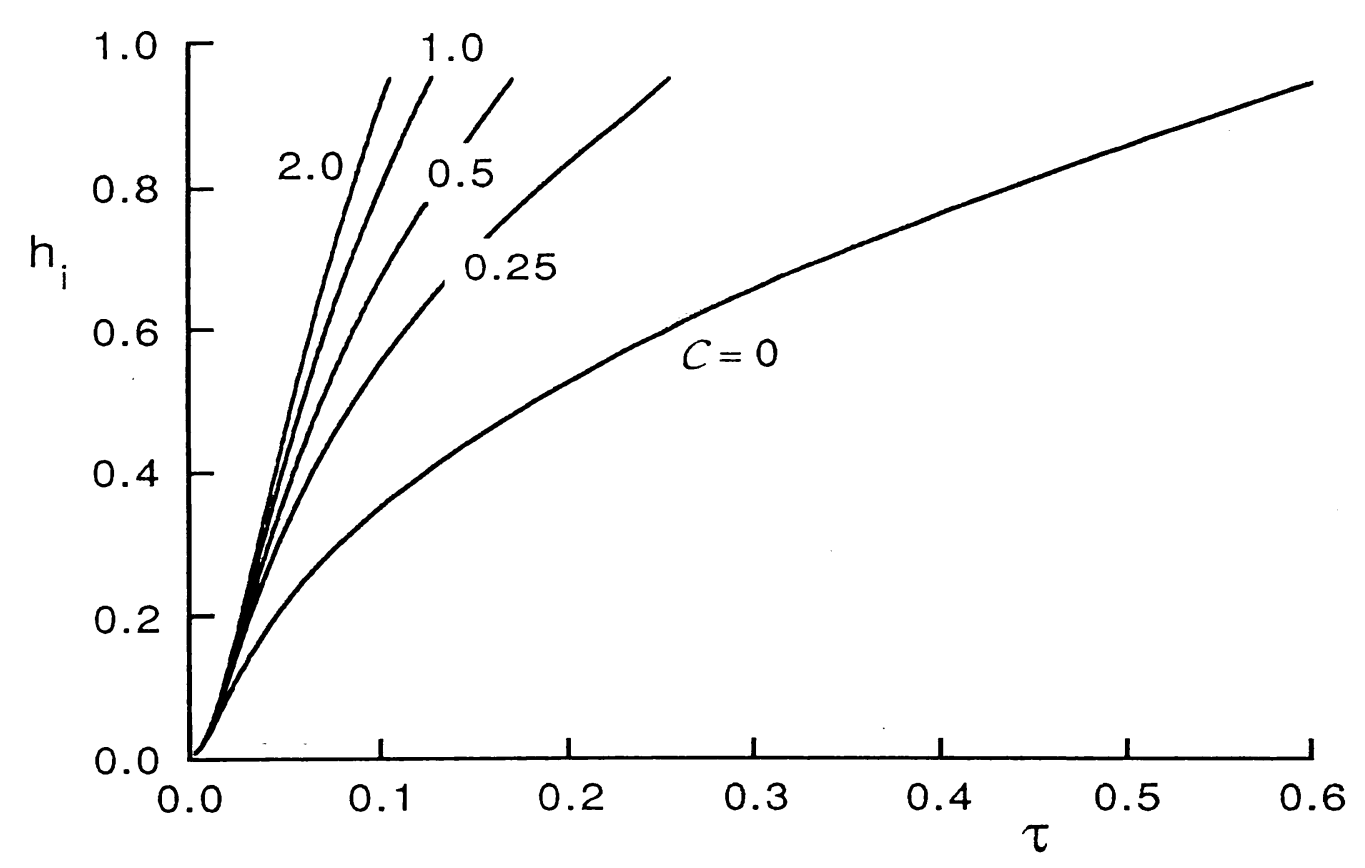

Figure 8: The dimensionless depth $h_{i}$ of a mushy layer grown at thermodynamic equilibrium as a function of time $\tau$ for various values of the concentration ratio $\mathcal{C}$. The other dimensionless parameter values used were $\mathcal{S}=1, \tilde{\mathcal{N}}=100$, and $\theta_{0}=1$. Note that, as $\mathcal{C}$ increases, the solid fraction in the mushy layer decreases, which allows the depth of the mushy layer to grow more rapidly.

within the mushy layer. The value of $\mathcal{C}$ is zero for a pure melt and is always positive otherwise. In this system of equations, the Stefan number $\mathcal{S}=\mathcal{L} / c_{m}\left(T_{L}\left(C_{0}\right)-T_{A}\right)$ only appears divided by $\mathcal{C}$, so we can deduce that positive values of $\mathcal{C}$ serve to reduce the effective value of the Stefan number. This is because, as seen from equation (3.8c), the solid fraction in the mushy layer decreases as $\mathcal{C}$ increases, so less latent heat is required to be removed in order to increase the depth of the mushy layer. This is the principal effect of composition on the solidification of a multi-component melt and it causes the mush-liquid interface to advance more rapidly than the solid-liquid interface in a pure melt. This is illustrated by the results presented in figure 8 . Note that increasing $\mathcal{C}$ produces similar results to those obtained by decreasing $\mathcal{S}[5]$.

A consequence of the fact that the release of latent heat is distributed throughout a mushy layer, rather than just at the mush-liquid interface, is that the rate of advance of the interface is controlled to a greater extent by a balance between the heat conducted through the mushy layer and the heat flux from the melt. Indeed, equations (3.7)-(3.11) predict an infinite rate of advance of the mush-liquid interface $\dot{h}_{i}$ when the superheat $\theta_{0}$ is zero [11] so that there is no convective flux from the melt. In general, the decaying superheat of the region of melt can cause the interface to advance very much faster than it would if latent heat were released there. However, this runaway behaviour is associated with very small values of the solid fraction in the regions of the mushy layer nearest the melt [12] and one must question whether such regions will remain mechanically robust. In addition, we shall see in section 4 that kinetic effects at the mush-liquid interface restrict this runaway behaviour by causing non-zero values of the solid fraction at the mush-liquid interface. 
Numerical solutions of the equations presented in this section have been found to agree very well with the results of laboratory experiments [5] in which mushy layers of solid ice were formed from aqueous solutions of isopropanol. This agreement, which can be seen in figure 9, gives support to the various approximations invoked in the development of the model. However, these same authors found that the region of melt became supersaturated during the course of the experiments (see figure $9 \mathrm{~b}$ ), an observation that is inexplicable by a theory employing the condition of equilibrium thermodynamics expressed by equation (3.1). Just as in section 2, the convection associated with equilibrium solidification at the roof can only reduce the superheat to small values; the melt cannot thereby be cooled below its liquidus temperature.

4. Kinetic effects. Mathematical models of convection driven by solidification that assume equilibrium thermodynamics, such as the models outlined in the previous two sections, show that the temperature of the melt decays towards the equilibrium solidification temperature (liquidus temperature) that is assumed to pertain to the solidification front. Convection ceases at some small value $\Delta T_{c}$ of the superheat, which is defined by the criterion that the Rayleigh number based upon that temperature difference and the full depth of the melt is less than its critical value. Brandeis and Marsh [13] coined the phrase 'convective liquidus' to apply to the temperature that the melt has when this occurs. They found experimentally that the 'convective liquidus' is almost equal to the actual liquidus temperature of the melt. Indeed, a simple scaling analysis shows that $\Delta T_{c}$ is given by

$$
\Delta T_{c} \sim R a_{c} \frac{\kappa \nu}{\alpha g H^{3}},
$$

where $R a_{c}$ is the critical value of the Rayleigh number $\left(R a_{c} \approx 10^{3}\right)$. This temperature difference is extremely small in magma chambers owing to the very large typical values of their depth $H$. For the parameter values in Table $1, \Delta T_{c} \approx 10^{-9}{ }^{\circ} \mathrm{C}$.

$\begin{array}{lll}\text { Quantity } & \text { Value } & \text { Units } \\ \kappa & 8 \times 10^{-3} & \mathrm{~cm}^{2} \mathrm{~s}^{-1} \\ \mu & 26 & \text { poise } \\ \nu & 10 & \mathrm{~cm}^{2} \mathrm{~s}^{-1} \\ \alpha & 10^{-4} & { }^{\circ} \mathrm{C}^{-1} \\ g & 10^{3} & \mathrm{~cm} \mathrm{~s}^{-2} \\ H & 10^{4} & \mathrm{~cm}\end{array}$

Table 1. Parameter values corresponding approximately to those appropriate to a diopside melt in a layer $100 \mathrm{~m}$ deep.

However, the assumption of equilibrium thermodynamics is only an approximation; the actual temperature of an advancing phase boundary must always be slightly below the equilibrium liquidus temperature of the melt in order for the crystal to 

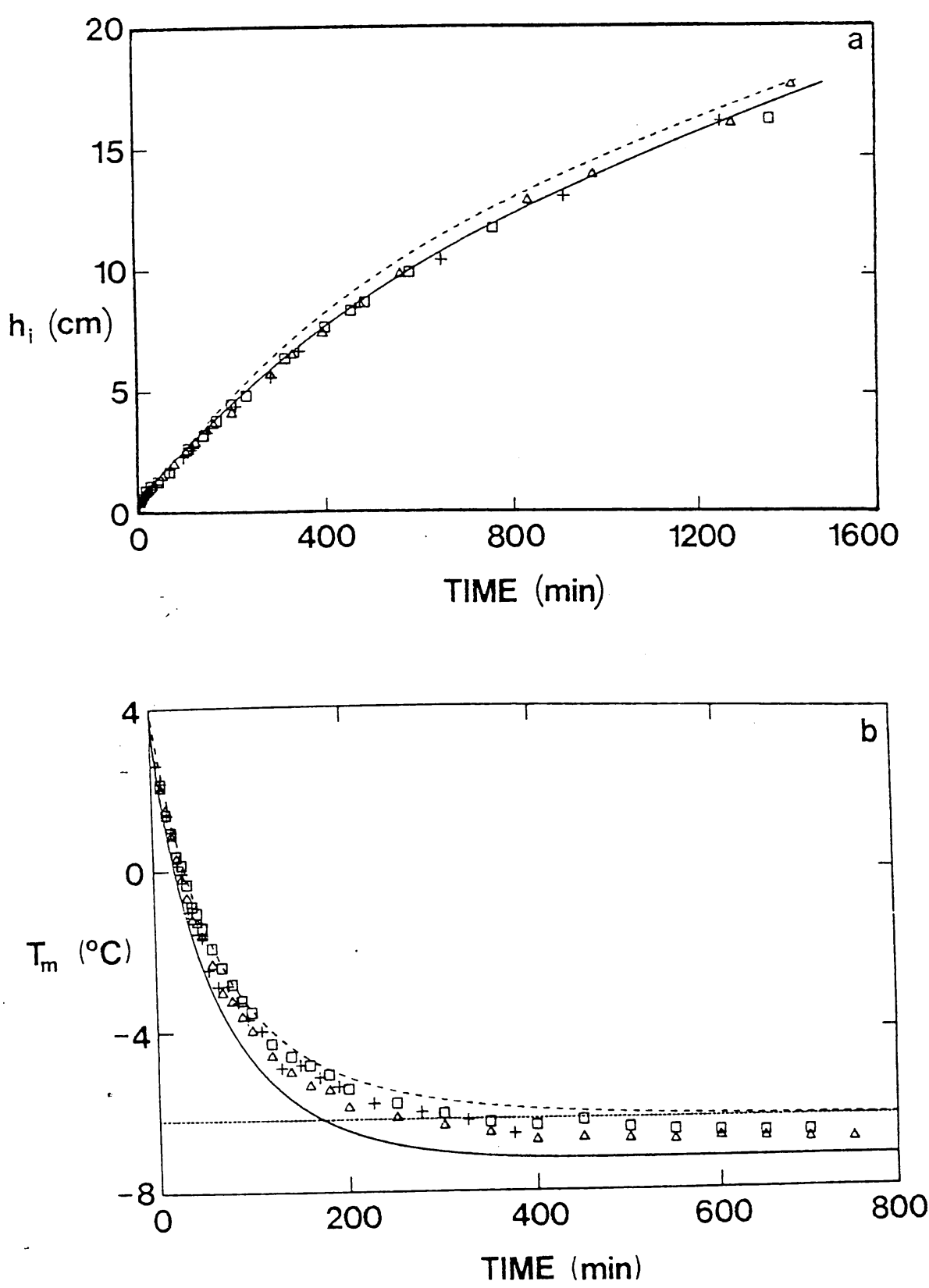

Figure 9: Comparisons of theoretical predictions with results of experiments on the solidification of ice from a mixture of water and isopropanol [5]. The dashed curves are the predictions of the model that assumes equilibrium thermodynamics, while the solid curves are predictions of the model that includes kinetic undercooling at the mush-liquid interface. Excellent agreement between theory and experiment is found for the depth of the mushy layer (figure a), and the theory successfully accounts for the observed supersaturation of the melt (figure b). Note that heat transfer from the laboratory may have caused the experimental data of temperature to be slightly higher than they would have been had the system been perfectly insulated. 
grow [14]. This undercooling, $\Delta T_{k}$, is a function of the rate of advance of the interface. So far as describing convection in the melt is concerned, the approximation of equilibrium thermodynamics is only good while the superheat is much larger than the kinetic undercooling $\Delta T_{k}$. However, we shall see in section 5 that $\Delta T_{k}$ is typically much larger than $\Delta T_{c}$, so that the concept of the 'convective liquidus' rarely applies, and convection in magma chambers continues until the depth of remaining melt is very small, even in cases in which there is no initial superheat.

The relationship between the kinetic undercooling and the rate of advance of the solidification front varies with the material that is being solidified and the molecular nature of the solidification process. For a solid that grows by the method of continuous growth [15], the relationship is linear of the form

$$
\dot{h}_{i}=\mathcal{G}\left(T_{L}-T_{i}\right)
$$

where $\mathcal{G}$ is a constant, $T_{L}$ is the liquidus temperature of the melt and $T_{i}$ is the temperature at the solidification front.

Kerr et al. [6] conducted experiments to verify equation (4.2) for the case of ice growing from a mixture of water and isopropanol. They found good agreement with a linear law for undercoolings up to about $3^{\circ} \mathrm{C}$ and determined the constant $\mathcal{G}$ to be approximately $2.2 \times 10^{-4} \mathrm{~cm} \mathrm{~s}^{-1}{ }^{\circ} \mathrm{C}^{-1}$ for this system. They proceeded to incorporate the kinetic growth law (4.2) into the general model for the growth of mushy layers as follows.

A mathematical model for the solidification of a melt cooled from above that takes account of the kinetics of crystallization follows that presented in section 3 very closely, with equation (3.1) replaced by equation (4.2). The dimensionless system of governing equations and boundary conditions is given by (3.7)-(3.11) with the boundary condition $(3.7 \mathrm{c})$ replaced by

$$
\theta=\theta_{i} \quad\left(z=h_{i}\right)
$$

and equations (3.9) and (3.10) replaced by

$$
\left(\mathcal{S} \phi_{i}+0_{m}-0_{i}\right) \dot{h}_{i}=\left.\frac{\partial \theta}{\partial z}\right|_{h_{i}-}-\tilde{\mathcal{N}}\left(\theta_{m}-\theta_{i}\right)^{\frac{4}{3}}
$$

$$
\left(1-h_{i}\right) \dot{\theta}_{m}=-\tilde{\mathcal{N}}\left(\theta_{m}-\theta_{i}\right)^{\frac{4}{3}}
$$

together with the dimensionless kinetic growth law

$$
\dot{h}_{i}=-\gamma \theta_{i}
$$

Note that, although kinetic undercooling is included at the mush-liquid interface (equation (4.6)) it is still neglected in the interior of the mushy layer since the very large specific surface area of solid-liquid interfaces in the interior allows for very rapid relaxation to equilibrium there. 
These equations are solved by treating equation (4.4), with $\dot{h}_{i}$ replaced by the right-hand side of (4.6), as an algebraic equation for the unknown interface temperature $\theta_{i}$ and treating equation (4.6) as an evolution equation for $h_{i}$. Note that, since the interfacial temperature $T_{i}$ is no longer zero, the solid fraction at the interface is also non zero, and there is consequently a contribution to the thermal balance at the interface (4.4) due to latent heat.

The incorporation of the kinetic growth law into the theoretical model makes only a small correction to the prediction of the growth of solid from the cooled roof, which is in excellent agreement with the experimental results of Kerr et al. [6], as can be seen in figure 9a. Much more importantly, the model now predicts the observed supersaturation of the melt that occurs after a finite time in experiments in which the melt is initially undersaturated (figure $9 b$ ).

Apart from maintaining convection, this phenomenon has important consequences for the formation of solid from the melt. Once the melt is supersaturated (supercooled), any nucleation sites within the body of the melt provide locations for further solidification of the melt, regardless of their position relative to the cooled boundary. This was seen dramatically in experiments in which sodium sulphate was crystallized from its aqueous solution $[6,16]$; crystal growth took place at the floor of the experimental tank, as well as near the roof of the tank, even though the system was cooled only at the roof.

As crystals grow in the interior of the melt, latent heat and residual liquid are released. The latent heat raises the temperature of the supercooled melt, while the residual liquid, which is of a lower concentration, depresses the liquidus temperature of the melt. These effects combine to cause the level of supercooling to be reduced. The crystals at the floor in the laboratory experiments were observed to have a very large surface area exposed to the melt, and this surface area would be even larger if, for example, the crystals were to grow in suspension. In addition, since the residual fluid is buoyant and rises convectively away from the crystals, the internal growth of crystals is very efficient both at reducing the level of supersaturation and in keeping the melt well mixed. A good approximation to the rate of internal crystal growth can therefore be made by assuming that it is sufficiently rapid to keep the melt close to equilibrium. We therefore assume that the uniform temperature $T_{m}$ and composition $C_{m}$ of the melt are related by equation (3.1), while conservation of solute demands that the composition of the melt is given by

$$
\left(C_{s}-C_{m}\right) \dot{h}_{f}=\left(H-h_{i}-h_{f}\right) \dot{C}_{m},
$$

where $h_{f}$ is the depth that the crystals would occupy if they were to form a solid layer at the floor of the chamber. Note, however, that the mathematical description takes no explicit account of where the internal crystal growth takes place and therefore equation (4.7) is strictly a prediction for the volume of crystals grown, with the interpretation that $h_{f}$ is the volume per unit horizontal area.

As the composition of the melt changes due to the internal growth of crystals, so the composition of the liquid incorporated into the mushy layer also changes. The 
the floor, with the accompanying latent heat that is released being transported by convection across the fluid layer and into the mushy layer. With these fundamental concepts in mind, we can make some quantitative calculations appropriate to the cooling and solidification that occurs in magma chambers. Our desire to carry out such calculations acted as one of the major motivations behind the research.

Magma chambers come in a variety of shapes and sizes (see, for example, $[17,18,19,20,21]$. Many, however, are believed to have a horizontal scale which is considerably in excess of the vertical scale. This suggests that for these chambers the two- (and possibly three-) dimensional effects which occur at the ends of the chamber will be much less pronounced than those which occur at the floor and roof. We shall consider these to be horizontal so that a one-dimensional problem results. However, there may well be further details involved due to the irregular shape of the roof and floor of all magma chambers, though this is beyond our present analysis. We shall consider the base of the chamber to be insulated, though this too is an approximation, and note simply that any heat lost through the floor will only enhance the effects discussed in this section. Some investigations of the purely thermal aspects of this problem have been considered in $[22,23]$. At the top of the chamber, heat will be transferred by pure conduction into the (supposedly infinite) country rocks above. This necessitates coupling the partial differential equation of thermal diffusion to the heat flux through the composite layer at the roof, as is described quantitatively below.

Magma is a very complicated, multi-component crystallizing silicate melt. The composition of magma varies significantly with location within the earth and few concise measurements of physical and chemical properties exist. A simplified system, which has been studied considerably by geologists in the past, consists of a melt of anorthite and diopside for which there are well-documented values for thermochemical properties [24] and kinetic growth rates [25] and for which the phase diagram is reproduced in figure 7 . We shall confine our analysis to this particular system. We concentrate on anorthite-diopside systems with more diopside than the eutectic value, $58 \%$, which, above the eutectic temperature, cool to form solid diopside and release a less dense (diopside-depleted) melt.

We shall assume that an appropriate anorthite-diopside melt, at its liquidus temperature, is instantaneously emplaced in the chamber. The fact that the melt is at its liquidus temperature, or in other words that there is initially zero superheat in the system, is in accord with the standard geologists' view of silicate melts within the earth. That the melt be emplaced instantaneously is an oft-used theoretical simplification. To our knowledge there has been no theoretical analysis of the effects due to a continual input of magma, and incleed we know of only one experimental investigation [26] of such a situation.

With these assumptions on the physical aspects of the system to be studied in mind, we are ready to consider the quantitative analysis of the problem. This will demonstrate that even with no initial superheat whatsoever, the kinetic undercooling is sufficient to drive vigorous convective motions in the melt. Further, this leads to solidification at the floor, even though there is cooling only at the roof, in agreement 
The value of $\mathcal{G}$ is approximately $5.6 \times 10^{-5}$ poise $\mathrm{cm} \mathrm{s}^{-1}{ }^{\circ} \mathrm{C}^{-2}$ for diopside [25], so a typical value of $\Delta T_{k}$ is about $0.6^{\circ} \mathrm{C}$ (using the values in Table 1 ). Convection will continue until $\Delta T_{k}$ is about equal to the critical undercooling $\Delta T_{c}$ given by equation (4.1), that is until the depth of the remaining melt $h$ is about that given by

$$
\frac{h}{H} \sim\left(R a_{c}^{2} \frac{\kappa \nu^{2} \mathcal{G}}{\alpha^{2} g^{2} \mu H^{5}}\right)^{\frac{1}{6}} \text {. }
$$

This ratio is typically very small given the large dimensions of most magma chambers. It is therefore appropriate to model the evolution of a magma chamber using the assumption of vigorous convection of the melt driven solely by the small kinetic undercooling in cases when there is no initial superheat.

The governing equations in the composite layer, mushy layer, convecting melt and floor layer are given by (3.1)-(4.10) with the kinetic growth law (4.2) replaced by (5.1). The dynamic viscosity $\mu$ of the molten diopside, which varies with the temperature of the melt, can be represented by

$$
\mu=\exp \left[\left(12-52.5 x+62.5 x^{2}\right) \ln 10\right],
$$

where $x=1-1000 T^{-1}$ and $T$ is the absolute temperature of the melt (in Kelvin), a relationship obtained [27] by curve fitting the graphical data presented by Kirkpatrick [28].

Since heat is conducted into the overlying country rock, the temperature at the upper boundary of the magma chamber $T_{A}$ is no longer constant. It can be found in terms of a Green's function solution [29] of the thermal diffusion equation in the country rock, and is given by

$$
\begin{aligned}
T_{A}(t)-T_{\infty} & =\frac{1}{k_{r}} \sqrt{\frac{\kappa_{r}}{\pi}} \int_{0}^{t} \frac{f(\tau)}{\sqrt{t-\tau}} d \tau \\
& =\frac{1}{k_{r}} \sqrt{\frac{\kappa_{r}}{\pi}}\left\{2 \sqrt{t} f(t)+\sqrt{t} \int_{0}^{1} \frac{f(t u)-f(t)}{\sqrt{1-u}} d u\right\},
\end{aligned}
$$

where $T_{\infty}$ is the far-field temperature in the country rock, subscript ' $r$ ' denotes properties of the country rock and

$$
f(t)=\left[k \frac{\partial T}{\partial z}\right]_{z=0+}
$$

is the heat flux through the boundary $z=0$ at time $t$, which is evaluated in terms of the temperature field and thermal properties of the solidifying roof below $z=0$. The second expression (5.5b) for $T_{A}$, in which the singularity in the Green's function has been removed, was used in the numerical scheme.

Some of the results of our numerical integrations of the governing differential equations described above are presented in figures $13-16^{3}$. The curves in figure 13

\footnotetext{
${ }^{3}$ Since publication of [27], we have found an error in the computer program used to calculate the results presented in that paper. The error produced results equivalent to giving a falsely low value to the conductivity of the country rock, and therefore cooling and solidification times were over predicted throughout, by about $40 \%$. None of the general conclusions of that paper are affected by the correction of this error. Figures 13-16 correspond to figures 2,3b, 4 and 6 of [27], and show calculations made using the corrected program.
} 


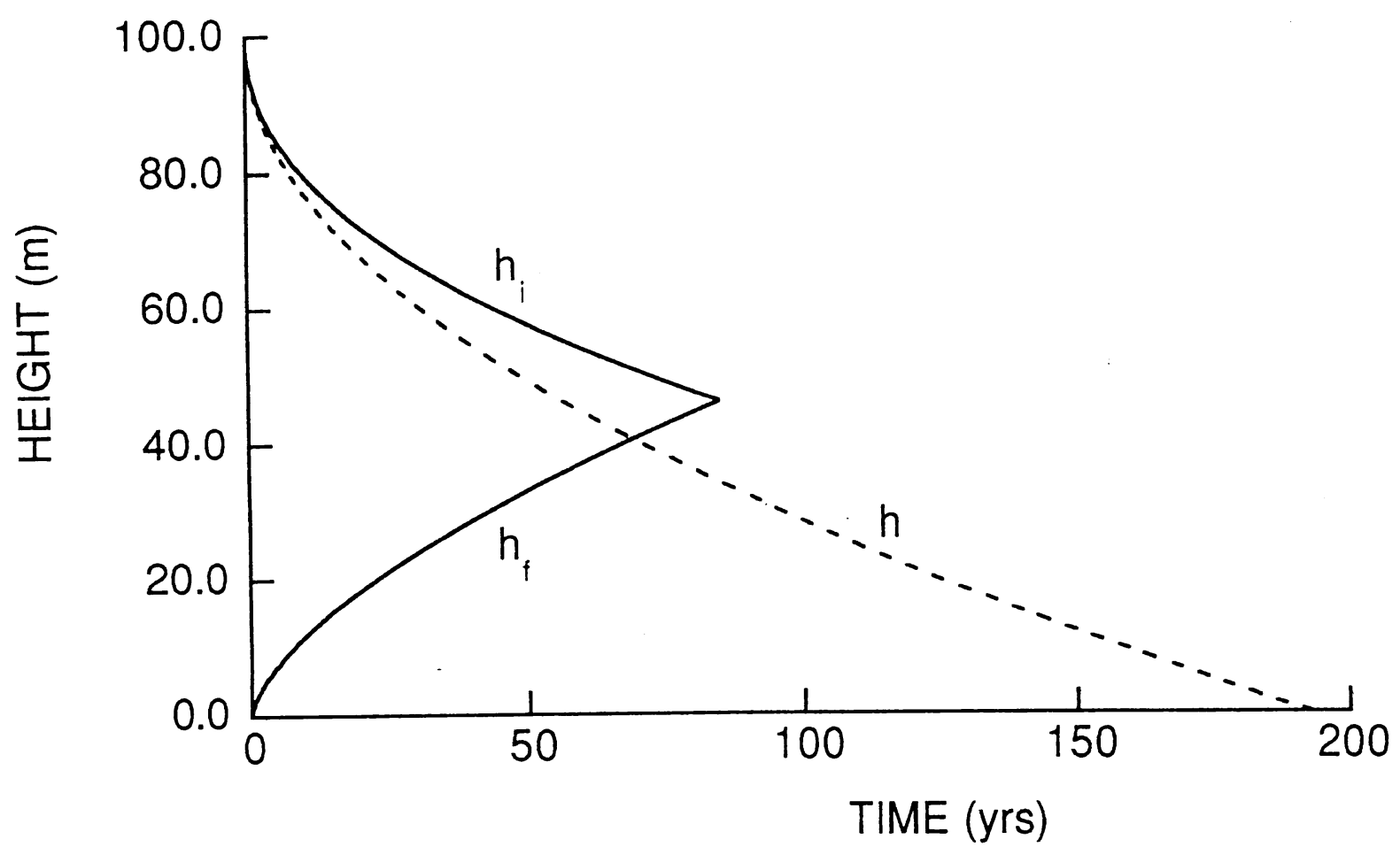

Figure 13: The predicted solidification of a layer of pure diopside cooled at its roof. The solid curves show the results of the model that includes convection and kinetic effects. The dashed curve shows, for the sake of comparison, the results of an unrealistic calculation in which convection is ignored.

depict as functions of time, the depth of solid grown at the roof and the floor from a layer of pure diopside initially $100 \mathrm{~m}$ thick emplaced into country rock at $0^{\circ} \mathrm{C}$. Also included for comparison is the resultant thickness of the roof layer if convective (and kinetic) effects are totally neglected, which means that no solid is grown at the floor. We see that the presence of convection reduces the time for complete solidification by about $56 \%$ over the physically (and geologically) inappropriate case when convection is neglected (c.f. Section 2). In addition convection allows approximately $45 \%$ of the chamber to solidify at the floor. The reason that convection decreases the time for complete solidification in this case is that it transports the latent heat of solidifcation very efficiently from the growth at the floor to the cooled roof. In contrast, when all the solidification takes place at the roof, all the latent heat must be conducted back through the much-thicker, growing crust.

The result of the calculations for an initial $100 \mathrm{~m}$ thick layer with a $20 \%$ anorthite composition is shown in figure 14 . Very rapidly after the initiation of the convective motions, a mushy layer develops beneath the composite solid layer near the roof, with a solid layer evolving from the floor. As the temperature in the melt decreases to the eutectic temperature $\left(1274^{\circ} \mathrm{C}\right)$, the mushy layer disappears, the layer growing from the roof becomes completely solid, and a composite layer of diopside and anorthite crystals begins to grow on the floor. With time a growing layer of eutectic composition ( $42 \%$ anorthite) extends into the remaining melt until total solidification has occurred.

The dimensionless time $\kappa t_{s} / H^{2}$ for complete solidification of a layer of initial 


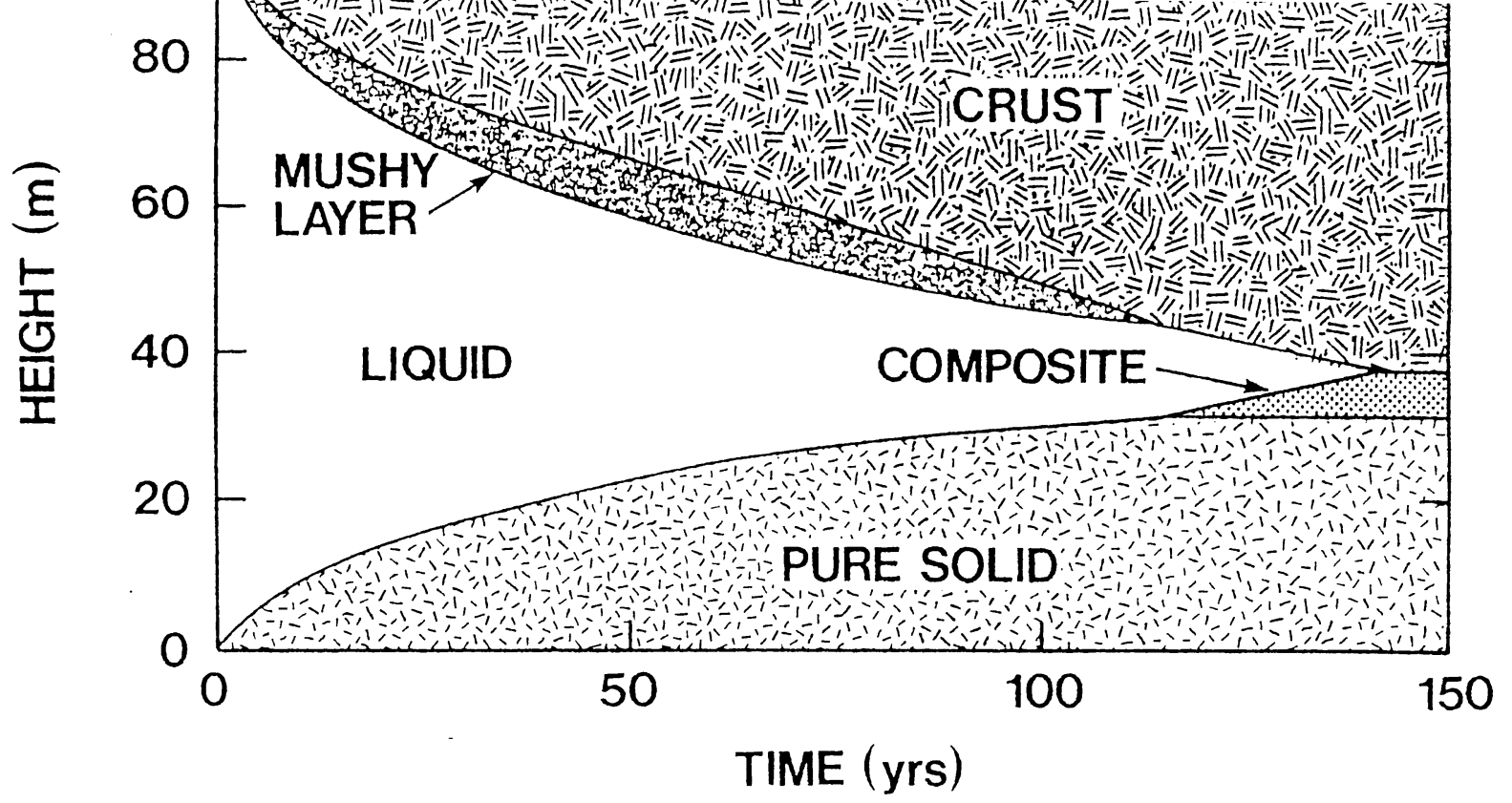

Figure 14: The predicted solidification of a layer of melt of composition $\mathrm{An}_{20} \mathrm{Di}_{80}$, one hundred meters thick emplaced at its liquidus temperature $1352^{\circ} \mathrm{C}$ into country rock at $0^{\circ} \mathrm{C}$.

depth $H$ is graphed in figure 15a The increasing importance of convection as the layer thickness increases is seen from the decreasing time for solidification to take place compared with the purely conductive timescale $H^{2} / \kappa$. Increasing the height $H$, and thus the intensity of the convective motions, also increases the fraction of solid deposited at the floor, as seen in figure $15 \mathrm{~b}$.

The gradual decrease in the temperature of the melt is presented in figure 16a. Because the melt is constrained to lie on the liquidus, the decreasing melt temperature is related to the decreasing diopside concentration. After about 180 years, the temperature of the melt has decreased to the eutectic temperature and it then remains at that value until solidification is complete. The temperature of the interface between the mushy layer and the melt, which is always less than the temperature of the melt itself, is also graphed in figure 16a with their difference presented in figure 16b. Note that, with typical geological parameter values, even though the depicted temperature differences are of order of only a few tenths of a degree centigrade, this leads to values of the Rayleigh number, shown in figure $16 \mathrm{c}$, which are sufficiently large for vigorous convective motions to occur until the layer has almost completely solidified.

6. Lava lakes. The foregoing analysis can also be used, with only minor alteration, to interpret the solidification history of lava lakes. The principal mathematical difference between the models for lava lakes and magma chambers is in the boundary condition that is applied at the upper boundary. Whereas for a magma chamber the upper boundary condition reflects the conduction into the country rock (equation (5.5)), the appropriate condition for lava lakes is that of constant temperature, since they lose heat very efficiently into the well-mixed air or water above them, which is at 

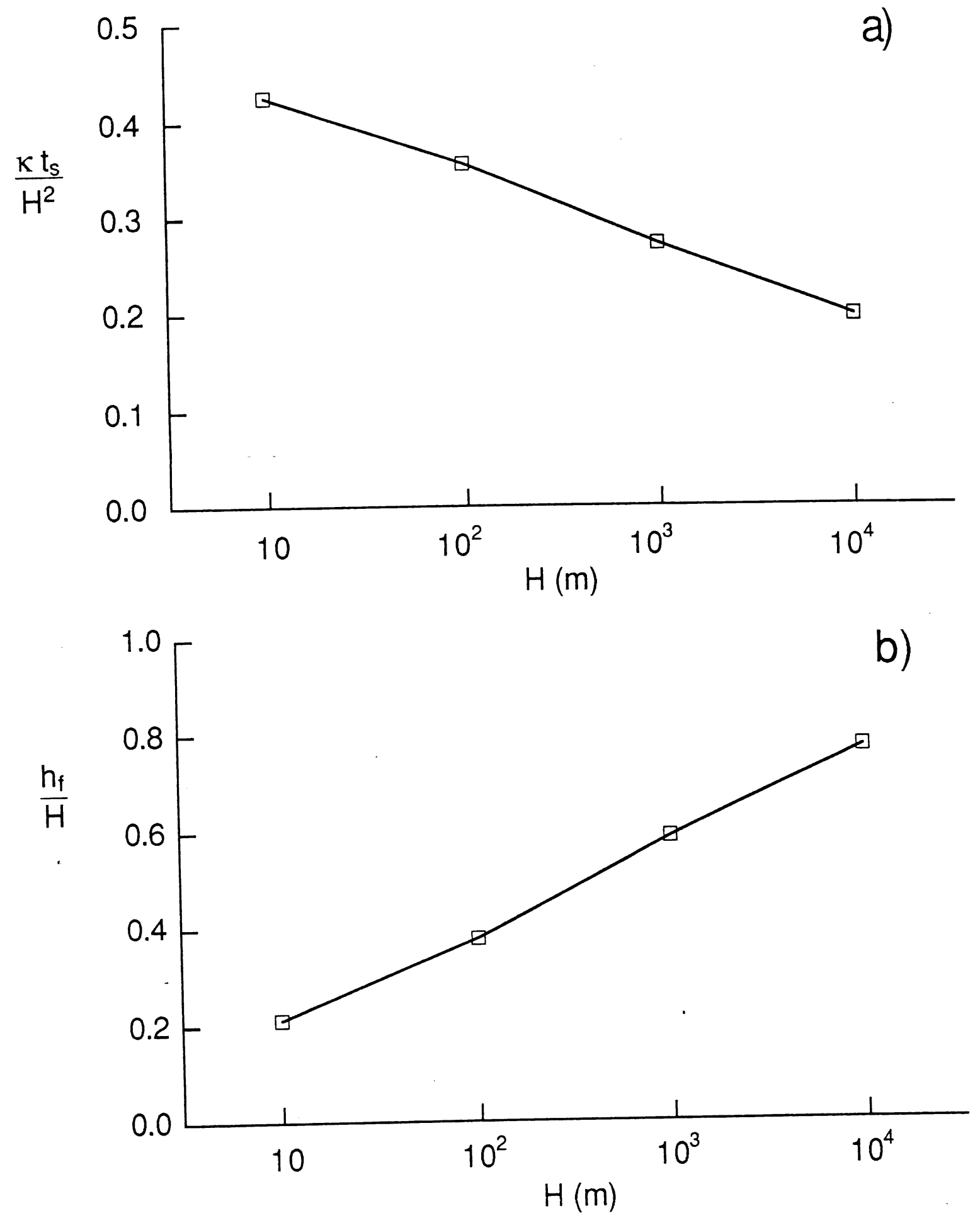

Figure 15: (a) The ratio of the time for complete solidification $t_{s}$ to the conductive timescale $H^{2} / r$ as a function of the initial depth $H$ of a layer of melt of composition $\mathrm{An}_{20} \mathrm{Di}_{80}$. As the depth increases, the vigour of convection increases and causes the solidification time to decrease relative to the conduction time. The stronger convection also causes the relative depth of crystals grown at the floor to increase, as shown in figure (b). 

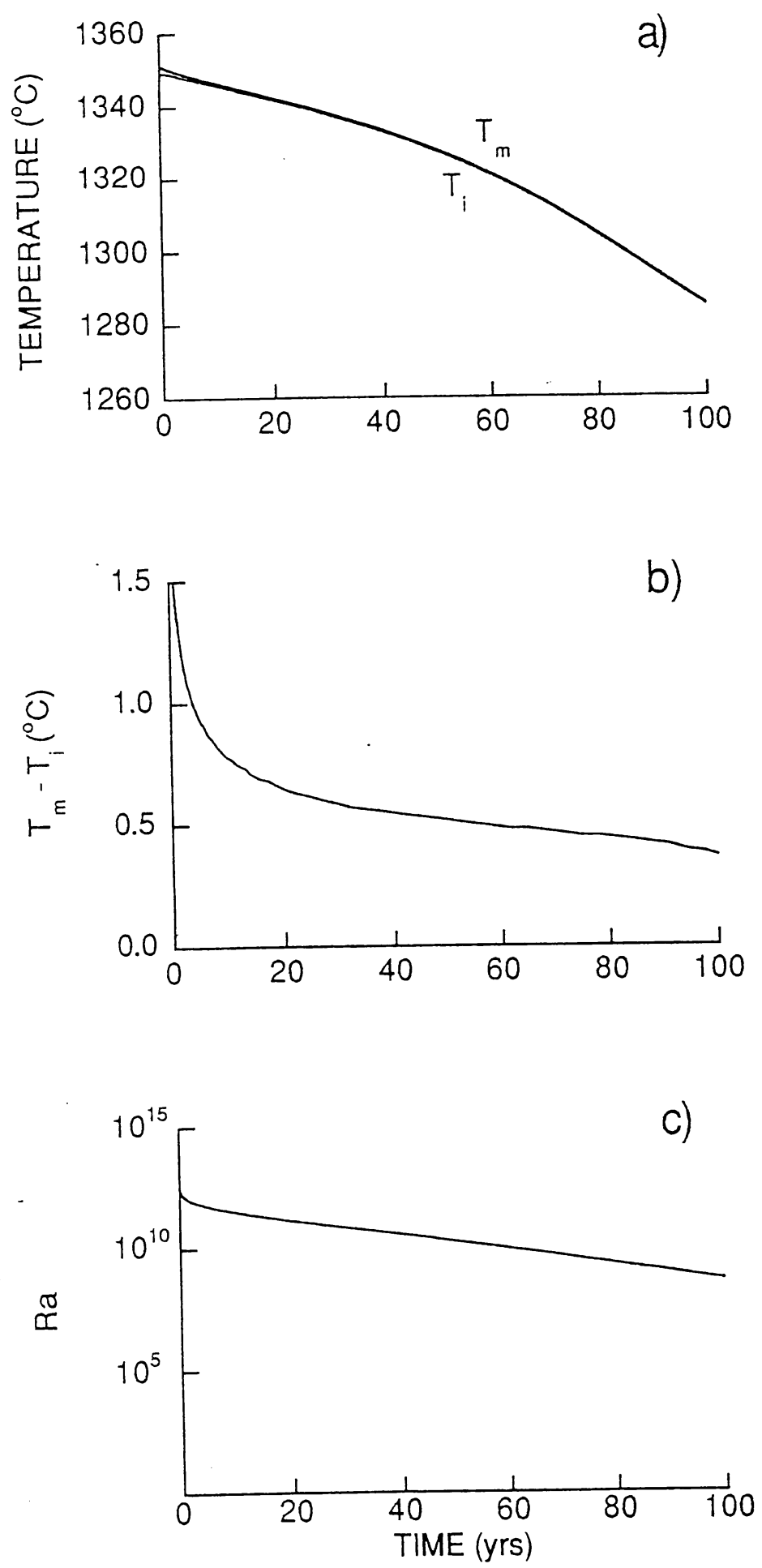

Figure 16: (a) The temperature of the melt $T_{m}$ and the temperature of the mushliquid interface $T_{i}$ as functions of time for a layer of melt of composition $\mathrm{An}_{20} \mathrm{Di}_{80}$ emplaced at its liquidus temperature $1352^{\circ} \mathrm{C}$ into country rock at $0^{\circ} \mathrm{C}$. Their difference is emphasized in (b). Although this temperature difference is only a fraction of a degree Centigrade, it is sufficient to give the very large Rayleigh numbers shown in (c). 
them, as we explain below.

The field data are for cases in which the lavas contained phenocrysts initially and hence were not superheated. These data are compared with a theoretical curve (labelled 'stagnant; latent heat; no overburden') derived from a purely-conductive model, which has the property that the interior temperature remains almost constant. Marsh contrasts this with the measurements of Jaupart et al. [22] (labelled 'JBA (1984)') and what is claimed to be a theoretical curve from Turner et al. [3] (labelled 'THS (1986)') which both show the temperature decreasing rapidly. However, both these studies correspond to systems that are initially superheated, and the results should correctly be represented on figure 17 of [30], which corresponds to our figure 18a, by curves starting above $T_{c}^{\prime}=1.0$ and decaying rapidly towards $T_{c}^{\prime}=1.0$. Such rapid decay is shown in figures $3 \mathrm{a}$ and $4 \mathrm{a}$ of section 2. Correct application of the model of Turner et al. [3] (presented in 2) to the case of a lava that is initially at its freezing temperature would predict that the interior temperature would remain constant at the freezing temperature $T_{c}^{\prime}=1.0$, and that there would be no convection.

In contrast, the model of Kerr et al. $[6,16]$ presented in sections 3 and 4, which includes kinetic effects, can be applied to the case of a lava lake that is not initially superheated and, in that case, predicts vigorous convection driven by kinetic undercooling and also predicts the dimensionless temperatures shown in figure 18b. These results, for lava lakes of different initial depths containing melts of composition $\mathrm{Di}_{80} \mathrm{An}_{20}$, show a slow decay of the interior temperature corresponding to the slow evolution of the composition of the magma as crystals of diopside form in the interior or at the base of the lake. Note that the calculations were stopped once the temperature reached the eutectic temperature of the $\mathrm{Di}-\mathrm{An}$ system.

These results, which are presented in terms of the same dimensionless variables used in [30], are in approximate agreement with the field data reproduced in figure 18a. This is also true of the purely conductive model. But, whereas Marsh [30] uses this agreement to argue against the existence of convection in magma chambers and lava lakes we have shown that these particular field data are equally consistent with a model that includes vigorous convection of the melt. Thus, given only the presented field data of temperature, it is not possible to decide between a model involving only conduction or one incorporating convective motions. However, if one also incorporates the well-established, fluid-mechanical principle that large Rayleigh numbers imply vigorous convection, then a distinction can be drawn. In addition, we believe that the field evidence for the convective model will be found in terms of the type of compositional segregation predicted and presented in figure 17, which cannot be explained simply in terms of conduction and phase equilibria.

7. Conclusion. This paper has discussed a series of one-dimensional mathematical problems that describe the cooling and resultant solidification of a oneor two-component melt. (A partial introduction to the associated two- and threedimensional problems is presented in two reviews by Huppert $[31,32])$. The two most 

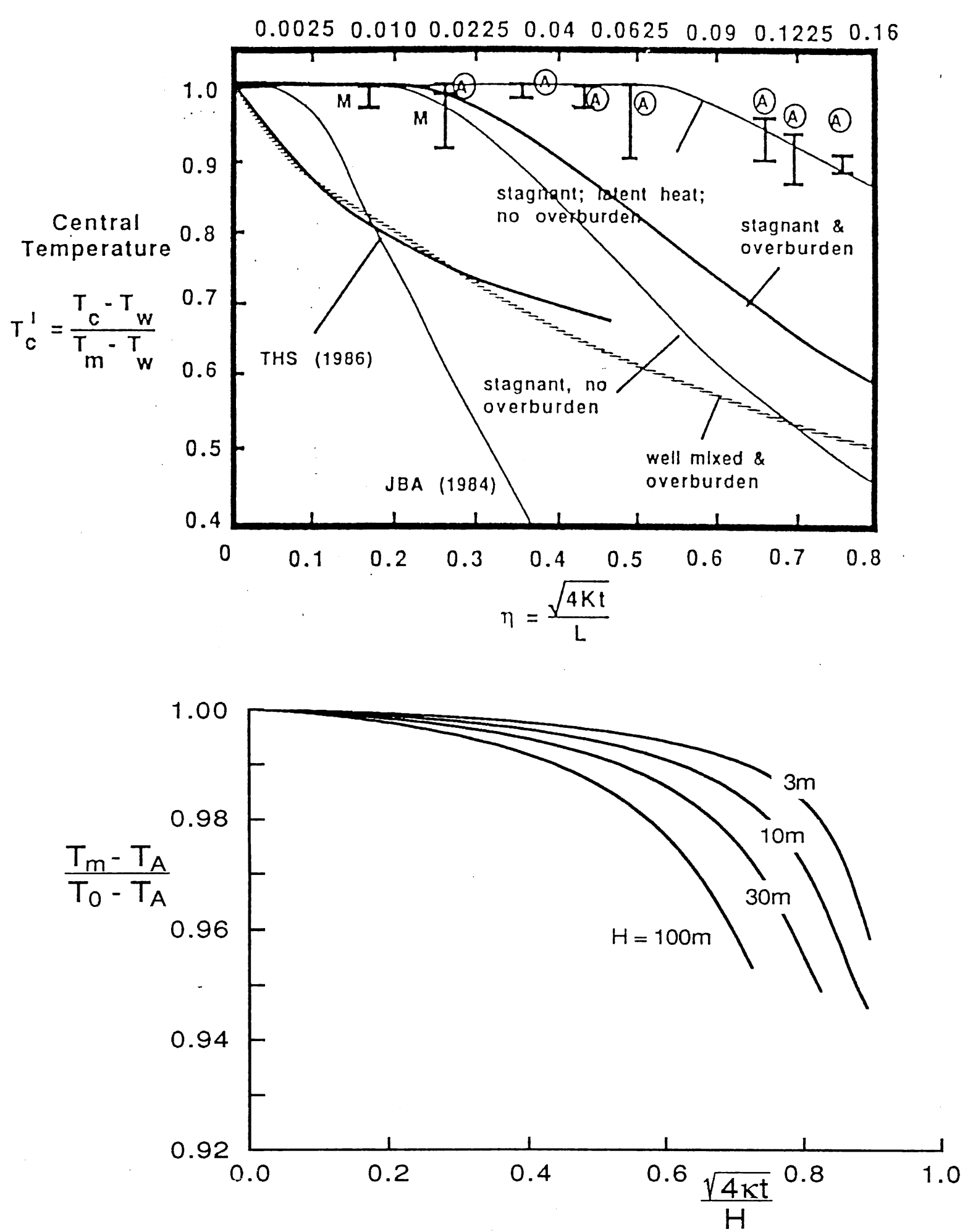

Figure 18: (a) A reproduction of figure 17 of [30], which includes some field data from lava lakes and a number of curves that are explained in the text. (b) The predicted interior temperature of lava lakes of different initial depths according to the model presented in the present paper. The scaling used in this figure is the same as that used in (a) above. The calculations used parameter values appropriate to a melt of composition $\mathrm{An}_{20} \mathrm{Di}_{80}$ so no direct quantitative comparison with the field data should be made. 
important concepts that run through the paper are that the timescale for solidification in the described systems is given by the conductive time scale, while, at the same time, convective motions in the solidifying melt play an essential role. For example, the removal of superheat from the melt takes place on the much-more-rapid convective timescale, and the presence of convection driven by kinetic undercooling at the solidifying interface of a two-component melt allows cooling at the roof of a system to lead to solidification in the interior of the melt and also at the floor. From a quantitative point of view, the convective heat transfer decreases the actual time taken for complete solidification, as indicated for particular examples in figures 5,13 and 15. In addition, the combination of convection and kinetic undercooling results in the vertical differentiation of the solidified magma or lava, an effect that is predicted by the current model.

Many of the specific-results of our mathematical models are in excellent agreement with data obtained from laboratory experiments. In addition, the general results are in qualitative agreement with the somewhat limited data available from geological observations. Calculations that are based on the two-component anorthite-diopside system predict a compositional gradient in the final solidified product which is similar to that observed in sills and old magma chambers throughout the world. The extension of the calculations made in order to describe the evolution of a lava lake indicates that the fluid part of the lava is in vigorous motion, while the thickness of the solidifying crust gradually increases. The observed rate of increase of the thickness of this crust agrees well with the results predicted by the theoretical model.

Certainly, more research is still needed to explain many of the details of the evolution of magma chambers and lava lakes. We look forward to the introduction of new physical concepts, more powerful mathematical and numerical techniques and fresh geological data to unravel the many chaotic features of solidification problems in particular and geophysical problems in general.

8. Acknowledgments. We are grateful to our colleagues M.A. Hallworth, R.C. Kerr, R.S.J. Sparks, J.S. Turner and A.W. Woods for contributing to the research reported in this paper, and would like, in addition, to thank them, along with J.R. Lister, for their helpful comments on an earlier version of the manuscript. The research work of H.E. Huppert is supported by a grant from Venture Research International, ably directed by Don Braben. M.G. Worster is grateful for partial support from the Thermal Systems Program of the National Science Foundation.

\section{REFERENCES}

[1] J. Crank, Free- and Moving-Boundary Problems, Clarendon Press, Oxford, 1984.

[2] J. M. Hill, One-dimensional Stefan Problems: An Introduction, Longman, Harlow, 1987.

[3] J. S. Turner, H. E. IIuppert and R. S. J. Sparks, Fomatiites II: Experimental and theoretical investigations of post-emplaccment cooling and crystallization, J. Petrol., 27 (1986), pp. $397-437$. 
[4] R. C. Kerr, A. W. Woods, M. G. Worster and H. E. Huppert, Disequilibrium and macrosegregation during solidification of a binary melt, Nature, 340 (1989), pp. 357-362.

[5] R. C. Kerr, A. W. Woods, M. G. Worster and H. E. Huppert, Soldification of an alloy cooled from above. Part I Equilibrium growth, J. Fluid Mech., 216 (1990), pp.323-342.

[6] R.C. Kerr, A.W. Woods, M.G. Worster and H.E. Huppert, Solidification of an alloy cooled from above. Part II Non-equilibrium interfacial kinetics, J. Fluid Mech., 217 (1990), pp.331-348.

[7] J. S. Turner, Buoyancy Effects in. Fluids, Cambridge University Press, Cambridge, 1979.

[8] T. L. Wright and R.T. Okamura, Cooling and crystallization of tholeiitic basalt, 1965 Makaopuhi lava lake, Hawaii, U.S. Geol. Survey Prof. paper, 1004 (1977), 78pp.

[9] T. L. Wright, D. L. PECK and H. R. ShaW, Kílauea lava lakes: natural laboratories of study of cooling, crystallization and differentiation of basaltic magma in The Geophysics of the Pacific Ocean Basin and its Margin (G. H. Sutton, M. H. Manghnani and R. Moberly, Eds) American Geophysical Union, Washington D.C. (1976), pp. 375-390

[10] H.E. Huppert and M.G.Worster, Dynamic solidification of a binary melt, Nature, 314 (1985), pp. 703-707.

[11] M.G. Worster, Solidification of an alloy from a cooled boundary, J. Fluid Mech., 167 (1986), pp. 481-501.

[12] M.G. Worster, Natural convection in a mushy layer, J. Fluid Mech., 224 (1991), pp. 335-359.

[13] G. BRANDEIS AND B.D. MARSH The convective liquidus in a solidifying magma chamber: a fluid dynamic investigation, Nature, 339 (1989), pp.613-616.

[14] W. Kurz and D.J. Fisier Fundamentals of Solidification, Trans. Tech. Publications, Aedermannsdorf (1986)

[15] R.J. KirkPatrick, Crystal growth from the melt: a review, American Mineralogist, 60 (1975), pp.798-814.

[16] R.C. Kerr, A.W. Woods, M.G. Worster and H.E. Huppert, Solidification of an alloy cooled from above. Part III Stratification of the solid, J. Fluid Mech., 218 (1990), pp.337-354.

[17] M.P. RYan, R.Y. Koyanagi and R.S. Fiske, Modelling the three-dimensional structure of macroscopic magma transport systems: Application to Kilauea Volcano, Hawaii, J. Geophys. Res., 86 (1981), pp.7111-7129.

[18] R.S. Detrick, P. Buhl, E.Era, J Mutter, J. Orcutt, J. Madsen and T. Brocher, Multi-channel seismic imaging of a crustal magma chamber along the East Pacific Rise, Nature, 326 (1987), pp.34-41.

[19] K.C. Macdonald, Anatomy of the magma reservoir, Nature, 339 (1989), pp.178-179.

[20] M.S. Burnett, D.W. Caress and J.A. Orcutt, Tomographic image of the magma chamber at $12^{\circ} 50^{\prime} N$ on the East Pacific Rise, Nature, 339 (1989), pp.206-208.

[21] G.M. Kent, A.J. Harding and J.A. Orcutt, Evidence for a smaller magma chamber beneath the East Pacific Rise at $9^{\circ} 30^{\prime} N$ Nature, 344 (1990), pp.650-653.

[22] C. Jaupart, G. Brandeis and C.J. Allegre, Stagnant layers at the bottom of convecting magma chambers, Nature, 308 (1984), pp.535- 538.

[23] C. JaUpart AND G. BRANDEIS, The stagnant bottom layer of convecting magma chambers, Earth and Planet. Sci. Lett., 80 (1986) pp.183-199.

[24] D.F. Neill, R. Hon and A. Navrotsky, The igneous system $\mathrm{CaMgSi}_{2} \mathrm{O}_{6}-\mathrm{CaAl}_{2} \mathrm{Si}_{2} \mathrm{O}_{3}-$ $\mathrm{NaAlSi}_{3} \mathrm{O}_{5}$ : variations on a classic theme by Bowen, in Physics of Magmatic Processes (R.B. Hargraves, Ed), Princeton University Press, New Jersey, 1980. 


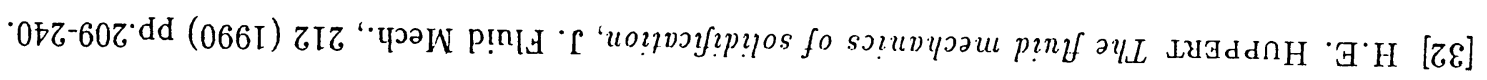

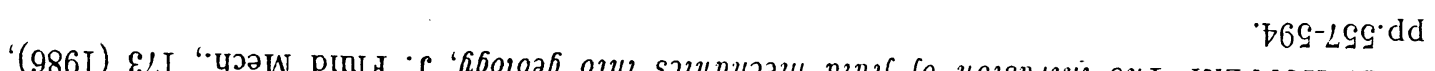

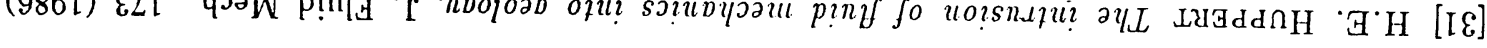
$\cdot 0 \varepsilon g-627 \cdot d d$ ' (686I) 0 \&

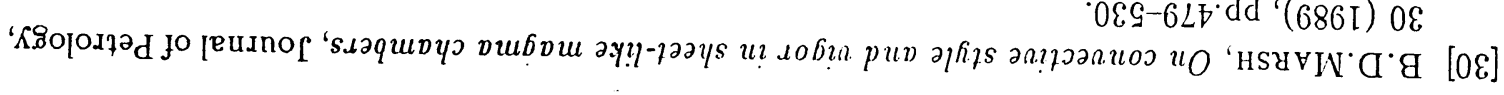

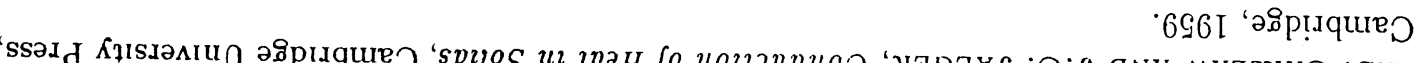

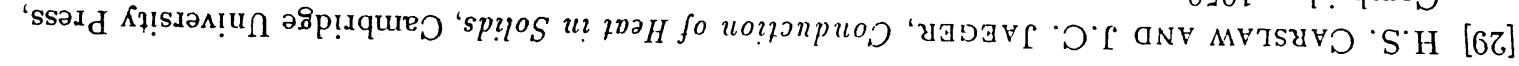

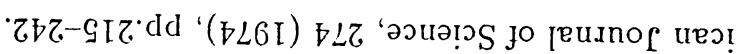

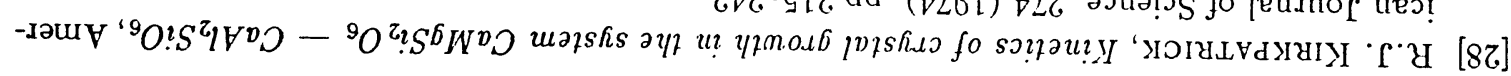

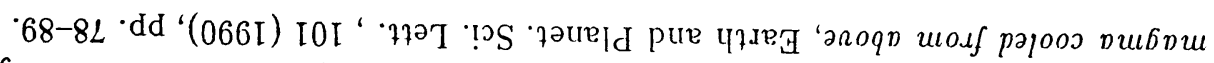

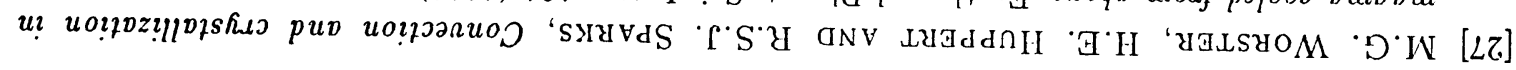

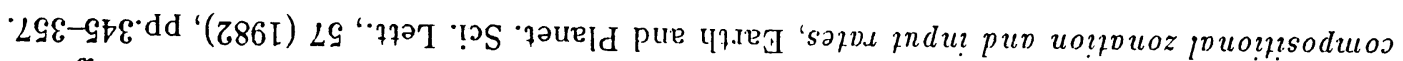

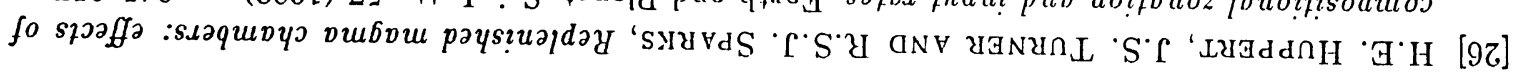

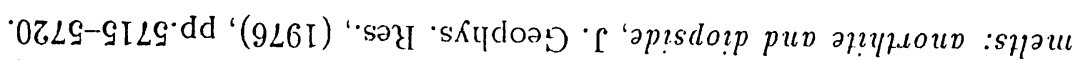

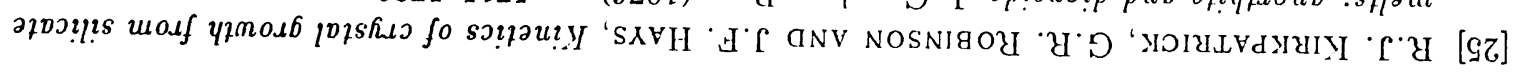




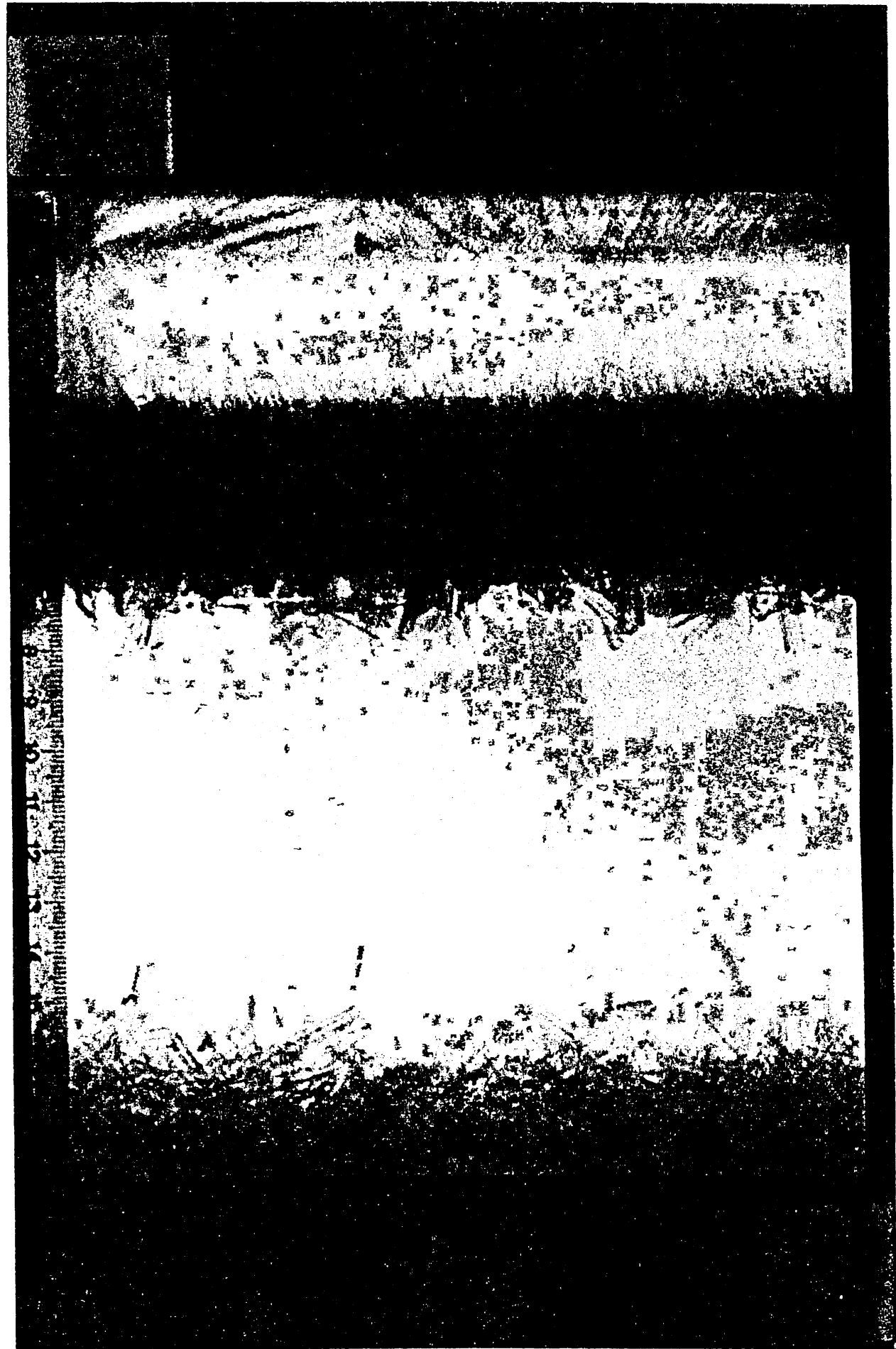

Figure 11: A photograph taken during an experiment in which an aqueous solution of $\mathrm{Na}_{2} \mathrm{SO}_{4}$ was cooled from above to form a composite layer next to the roof of the tank (the white region), a mushy layer below this and a layer of faceted $\mathrm{Na}_{2} \mathrm{SO}_{4} \cdot 10 \mathrm{H}_{2} \mathrm{O}$ crystals at the base. The experimental conditions were $C_{0}=16 \mathrm{wt} \% \mathrm{Na}_{2} \mathrm{SO}_{4}, T_{0}=$ $30.5^{\circ} \mathrm{C}, T_{B}=-17^{\circ} \mathrm{C}$, and $H=18.8 \mathrm{~cm}$. 


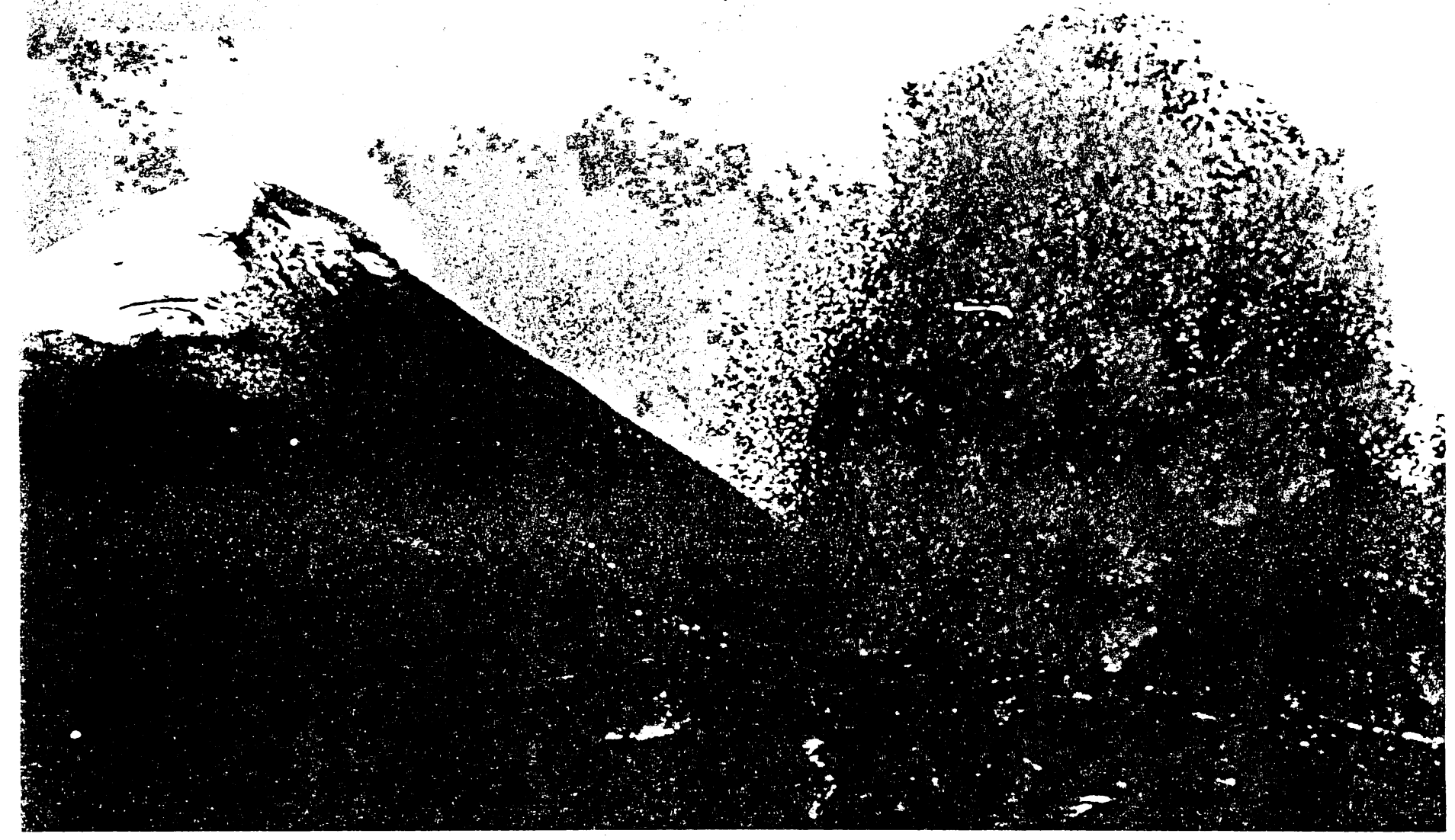

Figure 1: Lava fountaining during the eruption of Pu'u O'o, Hawaii, on 2 June 1986. The scale of the fountaining can be ascertained from the helicopter which appears as a mere speck two-thirds of the way up the fountain. Photograph by J. D. Griggs, United States Geological Survey. 
Stathis Filippas and Robert V. Kohn, Refined asymptotics for the blowup of $u_{t}-\Delta u=u^{p}$.

Patricia Bauman, Nicholas C. Owen and Daniel Phillips, Maximum principles and a priori estimates for an incompressible material in nonlinear elasticity

Patricia Bauman, Nicholas C. Owen and Daniel Phillips, Maximal smoothness of solutions to certain Euler-Lagrange equations from nonlinear elasticity

Jack Carr and Robert Pego, Self-similarity in a coarsening model in one dimension

J.M. Greenberg, The shock generation problem for a discrete gas with short range repulsive forces

George R. Sell and Mario Taboada, Local dissipativity and attractors for the Kuramoto-Sivashinsky equation in thin $2 \mathrm{D}$ domains

T. Subba Rao, Analysis of nonlinear time series (and chaos) by bispectral methods

Nicholas Baumann, Daniel D. Joseph, Paul Mohr and Yuriko Renardy, Vortex rings of one fluid in another free fall

Oscar Bruno, Avner Friedman and Fernando Reitich, Asymptotic behavior for a coalescence problem

Johannes C.C. Nitsche, Periodic surfaces which are extremal for energy functionals containing curvature functions

F. Abergel and J.L. Bona, A mathematical theory for viscous, free-surface flows over a perturbed plane Gunduz Caginalp and Xinfu Chen, Phase field equations in the singular limit of sharp interface problems Robert P. Gilbert and Yongzhi Xu, An inverse problem for harmonic acoustics in stratified oceans Roger Fosdick and Eric Volkmann, Normality and convexity of the yield surface in nonlinear plasticity H.S. Brown, I.G. Kevrekidis and M.S. Jolly, A minimal model for spatio-temporal patterns in thin film flow Chao-Nien Chen, On the uniqueness of solutions of some second order differential equations

Xinfu Chen and Avner Friedman, The thermistor problem for conductivity which vanishes at large temperature

Xinfu Chen and Avner Friedman, The thermistor problem with one-zero conductivity

E.G. Kalnins and W. Miller, Jr., Separation of variables for the Dirac equation in Kerr Newman space time

E. Knobloch, M.R.E. Proctor and N.O. Weiss, Finite-dimensional description of doubly diffusive convection

V.V. Pukhnachov, Mathematical model of natural convection under low gravity

M.C. Knaap, Existence and non-existence for quasi-linear elliptic equations with the p-laplacian involving critical Sobolev exponents

Stathis Filippas and Wenxiong Liu, On the blowup of multidimensional semilinear heat equations

A.M. Meirmanov, The Stefan problem with surface tension in the three dimensional case with spherical symmetry: non-existence of the classical solution

Bo Guan and Joel Spruck, Interior gradient estimates for solutions of prescribed curvature equations of parabolic type

Hi Jun Choe, Regularity for solutions of nonlinear variational inequalities with gradient constraints

Peter Shi and Yongzhi Xu, Quasistatic linear thermoelasticity on the unit disk

Satyanad Kichenassamy and Peter J. Olver, Existence and non-existence of solitary wave solutions to higher order model evolution equations

Dening Li, Regularity of solutions for a two-phase degenerate Stefan Problem

Marek Fila, Bernhard Kawohl and Howard A. Levine, Quenching for quasilinear equations

Yoshikazu Giga, Shun'ichi Goto and Hitoshi Ishii, Global existence of weak solutions for interface equations coupled with diffusion equations

Mark J. Friedman and Eusebius J. Doedel, Computational methods for global analysis of homoclinic and hetero clinic orbits: a case study

Mark J. Friedman, Numerical analysis and accurate computation of heteroclinic orbits in the case of center manifolds

Peter W. Bates and Songmu Zheng, Inertial manifolds and inertial sets for the phase-field equations

J. López Gómez, V. Márquez and N. Wolanski, Global behavior of positive solutions to a semilinear equation with a nonlinear flux condition

Xinfu Chen and Fahuai Yi, Regularity of the free boundary of a continuous casting problem

Eden, A., Foias, C., Nicolaenko, B. and Temam, R., Inertial sets for dissipative evolution equations Part I: Construction and applications

Jose-Francisco Rodrigues and Boris Zaltzman, On classical solutions of the two-phase steady-state Stefan problem in strips

Viorel Barbu and Srdjan Stojanovic, Controlling the free boundary of elliptic variational inequalities on a variable domain

Viorel Barbu and Srdjan Stojanovic, A variational approach to a free boundary problem arising in electrophotography

B.H. Gilding and R. Kersner, Diffusion-convection-reaction, free boundaries, and an integral equation

Shoshana Kamin, Lambertus A. Peletier and Juan Luis Vazquez, On the Barenblatt equation of elastoplastic filtration

Avner Friedman and Bei Hu, The Stefan problem with kinetic condition at the free boundary 
M.A. Grinfeld, The stress driven instabilities in crystals: mathematical models and physical manifestations

Bei Hu and Lihe Wang, A free boundary problem arising in electrophotography: solutions with connected toner region

Yongzhi Xu, T. Craig Poling, and Trent Brundage, Direct and inverse scattering of time harmonic acoustic waves in an inhomogeneous shallow ocean

Steven J. Altschuler, Singularities of the curve shrinking flow for space curves

Steven J. Altschuler and Matthew A. Grayson, Shortening space curves and flow through singularities

Tong Li, On the Riemann problem of a combustion model

L.A. Peletier \& W.C. Troy, Self-similar solutions for diffusion in semiconductors

C.J. van Duijn, L.A. Peletier \& R.J. Schotting, On the analysis of brine transport in porous media

Minkyu Kwak, Finite dimensional description of convective reaction-diffusion equations

Minkyu Kwak, Finite dimensional inertial forms for the 2D Navier-Stokes equations

Victor A. Galaktionov and Sergey A. Posashkov, On some monotonicity in time properties for a quasilinear parabolic equation with source

Victor A. Galaktionov, Remark on the fast diffusion equation in a ball

Hi Jun Choe and Lihe Wang, A regularity theory for degenerate vector valued variational inequalities

Vladimir I. Oliker and Nina N. Uraltseva, Evolution of nonparametric surfaces with speed depending on curvature, II. The mean curvature case.

S. Kamin and W. Liu, Large time behavior of a nonlinear diffusion equation with a source

Shoshana Kamin and Juan Luis Vazquez, Singular solutions of some nonlinear parabolic equations

Bernhard Kawohl and Robert Kersner, On degenerate diffusion with very strong absorption

Avner Friedman and Fernandor Reitich, Parameter identification in reaction-diffusion models

E.G. Kalnins, H.L. Manocha and Willard Miller, Jr., Models of $q$-algebra representations I. Tensor products of special unitary and oscillator algebras

Robert J. Sacker and George R. Sell, Dichotomies for linear evolutionary equations in Banach spaces

Oscar P. Bruno and Fernando Reitich, Numerical solution of diffraction problems: a method of variation of boundaries

Oscar P. Bruno and Fernando Reitich, Solution of a boundary value problem for Helmholtz equation via variation of the boundary into the complex domain

Victor A. Galaktionov and Juan L. Vazquez, Asymptotic behaviour for an equation of superslow diffusion. The Cauchy problem

Josephus Hulshof and Juan Luis Vazquez, The Dipole solution for the porous medium equation in several space dimensions

Shoshana Kamin and Juan Luis Vazquez, The propagation of turbulent bursts

Miguel Escobedo, Juan Luis Vazquez and Enrike Zuazua, Source-type solutions and asymptotic behaviour for a diffusion-convection equation

Marco Biroli and Umberto Mosco, Discontinuous media and Dirichlet forms of diffusion type

Stathis Filippas and Jong-Shenq Guo, Quenching profiles for one-dimensional semilinear heat equations

H. Scott Dumas, A Nekhoroshev-like theory of classical particle channeling in perfect crystals

R. Natalini and A. Tesei, On a class of perturbed conservation laws

Paul K. Newton and Shinya Watanabe, The geometry of nonlinear Schrödinger standing waves

S.S. Sritharan, On the nonsmooth verification technique for the dynamic programming of viscous flow

Mario Taboada and Yuncheng You, Global attractor, inertial manifolds and stabilization of nonlinear damped beam equations

Shigeru Sakaguchi, Critical points of solutions to the obstacle problem in the plane

F. Abergel, D. Hilhorst and F. Issard-Roch, On a dissolution-growth problem with surface tension in the neighborhood of a stationary solution

Erasmus Langer, Numerical simulation of MOS transistors

Hä̈m Brezis and Shoshana Kamin, Sublinear elliptic equations in $\mathbb{R}^{n}$

Johannes C.C. Nitsche, Boundary value problems for variational integrals involving surface curvatures

Chao-Nien Chen, Multiple solutions for a semilinear elliptic equation on $\mathbb{R}^{N}$ with nonlinear dependence on the gradient

D. Brochet, X. Chen and D. Hilhorst, Finite dimensional exponential atttractor for the phase field model

Joseph D. Fehribach, Mullins-Sekerka stability analysis for melting-freezing waves in helium-4

Walter Schempp, Quantum holography and neurocomputer architectures

D.V. Anosov, An introduction to Hilbert's 21st problem

Herbert E Huppert and M Grae Worster, Vigorous motions in magma chambers and lava lakes

Robert L. Pego and Michael I. Weinstein A class of eigenvalue problems, with applications to instability of solitary waves 